\title{
The Putative Protein Phosphatase MoYvh1 Functions Upstream of MoPdeH to Regulate the Development and Pathogenicity in Magnaporthe oryzae
}

\author{
Xinyu Liu, ${ }^{1}$ Bin Qian, ${ }^{1}$ Chuyun Gao, ${ }^{1}$ Shuohan Huang, ${ }^{2}$ Yongchao Cai, ${ }^{1}$ Haifeng Zhang, ${ }^{1}$ Xiaobo Zheng, ${ }^{1}$ \\ Ping Wang, ${ }^{3}$ and Zhengguang Zhang ${ }^{1}$ \\ ${ }^{1}$ Department of Plant Pathology, College of Plant Protection, Nanjing Agricultural University, and Key Laboratory of Integrated \\ Management of Crop Diseases and Pests, Ministry of Education, Nanjing 210095, China; ${ }^{2}$ Department of Pharmacy, Nanjing \\ Medical University, Nanjing 210029, China; and ${ }^{3}$ Department of Pediatrics, Louisiana State University Health Sciences Center, \\ New Orleans, LA 70118, U.S.A.
}

Submitted 23 November 2015. Accepted 15 March 2016.

\begin{abstract}
Protein phosphatases are critical regulators in eukaryotic cells. For example, the budding yeast Saccharomyces cerevisiae dual specificity protein phosphatase (DSP) ScYvh1 regulates growth, sporulation, and glycogen accumulation. Despite such importance, functions of Yvh1 proteins in filamentous fungi are not well understood. In this study, we characterized putative protein phosphatase MoYvh1, an Yvh1 homolog in the rice blast fungus Magnaporthe oryzae. Deletion of the MoYVH1 gene resulted in significant reductions in vegetative growth, conidial production, and virulence. The $\Delta M o y v h 1$ mutant also displayed defects in cell-wall integrity and was hyposensitive to the exogenous osmotic stress. Further examination revealed that the $\Delta$ Moyvh1 mutant had defects in appressorium function and invasive hyphae growth, resulting attenuated pathogenicity. Interestingly, we found that MoYvh1 affects the scavenging of host-derived reactive oxygen species that promotes $M$. oryzae infection. Finally, overexpression of the phosphodiesterase $M O P D E H$ suppressed the defects in conidia formation and pathogenicity of the $\Delta$ Moyvh1 mutant, suggesting MoYvh1 could regulate $M O P D E H$ for its function. Our study reveals not only the importance of MoYvh1 proteins in growth, differentiation, and virulence of the rice blast fungus but, also, a genetic link between MoYvh1 and $M o P D E H$-cAMP signaling in this fungus.
\end{abstract}

Eukaryotic cells utilize protein phosphorylation and dephosphorylation as one of the regulatory mechanisms for gene expression, signal transduction, and cell-cycle progression (Zolnierowicz and Bollen 2000). Protein phosphatases hydrolyze phosphorylated proteins resulting in dephosphorylation with consequent effects on downstream signaling pathways. Protein phosphatases are divided into three classes according to their substrates, protein tyrosine phosphatases that hydrolyze phosphotyrosine residues, protein serine/threonine phosphatases that hydrolyze phosphoserine/threonine residues, and dual-specificity protein phosphatases (DSPs) that hydrolyze phosphotyrosine and phosphoserine/threonine residues (Keyse 1995).

Corresponding author:Z.ZZhang; E-mail: zhgzhang @njau.edu.cn; Telephone: +86-25-84396972; Fax: +86-25-84395325

*The $\boldsymbol{e}$-Xtra logo stands for "electronic extra" and indicates that eleven supplementary figures and two supplementary tables are published online.

(c) 2016 The American Phytopathological Society
The first eukaryotic member of the DSP family, ScYvh1, was identified as a vaccinia virus VH1 phosphatase homolog in Saccharomyces cerevisiae (Guan et al. 1992). Alignment of YVH1 phosphatases from multiple species showed that all contain the conserved domain characteristic of DSPs in the $\mathrm{N}$-terminal. At the core of this domain, there is the HCXXGXXR sequence that represents the catalytic core of tryptophan phosphatases. And the essential cysteine of the sequence forms a thiophosphate intermediate in DSP-catalyzed dephosphorylation (Denu and Dixon 1995; Kumar et al. 2004). Substitution of this cysteine by serine abolished its hydrolytic activity in vitro (Alessi et al. 1993; Ishibashi et al. 1992; Sun et al. 1993) and function in vivo (Aroca et al. 1995; Doi et al. 1994; Sun et al. 1993). The Cterminal domain of Yvh1 is also conserved, containing seven invariant cysteines, and one histidine reminiscent of sequences represented the zinc-binding domain. The zinc-binding domain is known to mediate protein-protein or DNA-protein interactions. As demonstrated in budding yeast and in human fungal pathogens, the zinc finger proteins function in a wide range of processes, including amino acid and vitamin synthesis, carbon and nitrogen metabolism, meiosis, and morphogenesis (Fang et al. 2003; Liu and Chang 2009). Other roles of zinc-binding domain include regulation of genes involved in the stress response and pleiotropic drug resistance (MacPherson et al. 2006). Deletion of the $S c Y V H 1$ gene caused defects not only in vegetative growth but also in spore maturation, glycogen metabolism, and ribosome biogenesis (Beeser and Cooper 2000; Guan et al. 1992). In addition, the $S c Y V H 1$ transcript level increased significantly in response to nitrogen starvation at low temperatures (Sakumoto et al. 1999). In another report, the decreased sporulation of a $\Delta S c y v h 1$ strain was associated with the reduction in the transcription levels of a pair of sporulation-related genes (Ma et al. 1999). Moreover, an assay of the inhibitory effect to Congo Red (CR), which is a cell-wall stress agent, on ScYvh1 demonstrated that ScYvh1 functions in the maintenance of cellwall integrity (CWI) by influencing the Mck1-Mkk1-Mps1 mitogen-activated protein kinase (MAPK) pathway (SacristánReviriego et al. 2015).

Deletion of $Y V H 1$ also affected cyclic adenosine monophosphate (cAMP)-mediated signaling (Beeser and Cooper 2000). cAMP, a second messenger molecule produced in response to nutrients, is involved in the inhibition of downstream signaling components such as the phosphorylating enzyme protein kinase A (PKA) (Daniel et al. 1998). In fungi, the balance of intracellular cAMP levels is regulated by the activities of adenylyl cyclase and 
phosphodiesterase (PDEase). In $S$. cerevisiae, overexpression of ScPDE2, which encodes a high-affinity PDEase modulating the basal cAMP level, suppressed the defect in sporulation caused by deletion of ScYVH1 (Beeser and Cooper 2000). This suggests that ScYvh1 possibly functions upstream of the ScPde2-mediated cAMP pathway. In the opportunistic fungal pathogen Candida albicans, disruption of the CaYVHI gene resulted in slowed growth and altered cell morphology as well as virulence and CaYvh1 was also found to participate in nuclear division and septum formation (Hanaoka et al. 2005). Despite these findings, functions of Yvh1 proteins in filamentous fungi remain obscure.

Magnaporthe oryzae, the causal agent of rice blast disease, is widely used to study plant-pathogen interactions (Wilson and Talbot 2009). In this fungus, cAMP signaling is required for many aspects of development and pathogenicity. Deletion of $M o M A C 1$, which encodes adenylyl cyclase, resulted in a defect in virulence, and the defect could be suppressed by addition of exogenous cAMP or obliteration of the protein kinase PKA regulatory subunit. MoRgs1, one of the main regulators of G-protein signaling, also participates in the regulation of the cAMP pathway (Liu et al. 2007; Zhang et al. 2011b). Similarly, MoPdeH, a high-affinity PDEase, regulates conidia formation, pathogenicity, and invasive growth. Deletion of $M o P D E H$ not only increased the intracellular cAMP level but also reduced conidiation and virulence (Zhang et al. 2011a). Furthermore, recent studies reveal that MoPdeH interacts with MoMck1, which is one of the components of the MoMck1-MoMkk1MoMps1 MAPK cascade and plays critical roles for CWI and pathogenicity (Jeon et al. 2008; Xu et al. 1998). Overexpression of MoMCK1 could suppress defects in hyphal autolysis and pathogenicity of the $\triangle M o p d e H$ mutant that suggested that MoPdeH involves CWI and might be modulated by the MAPK cascade. Moreover, we found that MoMck1 regulates $M o P D E H$ expression through a feedback loop, to impact conidial development and pathogenicity through cellular cAMP levels (Yin et al. 2015).

In this study, we continued our studies by demonstrating that DSP MoYvh1 also plays an important role in vegetative growth, conidia formation, and CWI. MoYvh1 affects the turgor pressure in appressoria and scavenging of host-derived reactive oxygen species (ROS) to promote $M$. oryzae infection. Furthermore, overexpression of $M o P D E H$ suppressed the defects of the $\triangle M o y v h 1$ mutant in conidia formation and pathogenicity, indicating that MoYvh1 functions upstream of MoPdeH. Finally, we have analyzed MoYvh1 domain structures and showed that its $\mathrm{C}$-terminal zinc-binding domain is required for its characterized functions.

\section{RESULTS}

Identification of $\mathrm{MoYVH} 1$ from $\mathrm{M}$. oryzae and functional complementation of a $S$. cerevisiae $\Delta S c y v h 1$ mutant.

Examination of the $M$. oryzae genome database maintained at the Broad Institute revealed that MGG_09700 shares high amino acid sequence homology (29\% similarity) with $S$. cerevisiae ScYvh1, a member of the dual-specificity family of protein phosphatases, and we named it MoYvh1. MoYvh1 consists of 394 amino acids and contains a conserved tyrosine phosphatase catalytic domain within a dual-specificity phosphatase domain at its $\mathrm{N}$-terminus and a zinc-binding domain consisting of one histidine and eight cysteine residues at its $\mathrm{C}$-terminus (Supplementary Fig. S1A). Comparison of conserved tyrosine phosphatase catalytic domains in fungi showed that the MoYvh1 phosphatase catalytic domain contains an HCXXGXSR sequence similar to that found in other filamentous fungi. We expressed $M o Y V H 1$ in a $\triangle S c y v h 1$ mutant, using the yeast expression vector pYES2, and analyzed the transformants. The transformants showed suppressed defects in sensitivity to $\mathrm{CR}$, glycogen accumulation, cold sensitivity, and dityrosine production when compared with the original $\Delta S c y v h 1$ mutant (Beeser and Cooper 1999, 2000; Sacristán-Reviriego et al. 2015; Sakumoto et al. 1999), indicating that MoYvh1 functions heterologous in S. cerevisiae (Supplementary Fig. S2).

To further understand the functional roles of MoYvh1, we examined its transcript levels at various developmental stages. MoYVH1 transcript levels were relatively high from 24 to $36 \mathrm{~h}$ postinoculation (hpi) as determined by quantitative reverse transcription-polymerase chain reaction (qRT-PCR) (Supplementary Fig. S3). During these periods, MoYVH1 transcript levels were almost tenfold higher than those in conidia, indicating that $\mathrm{MoYVH} 1$ was highly expressed during infection.

\section{MoYvh1 is involved}

in vegetative growth and conidia formation.

Gene-targeted replacement was used to investigate the function of MoYvh1. Following the methods described by Zhang et al. (2009b) and following transformation, we generated and verified the $\Delta$ Moyvhhl deletion mutants (Supplementary Fig. S4).

We examined the vegetative growth of the $\Delta$ Moyvhl mutant on complete medium (CM), minimal medium, straw decoction and corn agar (SDC), and oatmeal medium plates. The $\Delta$ Moyvhl mutant exhibited significantly reduced vegetative growth after incubation at $28^{\circ} \mathrm{C}$ for 7 days in the dark (Fig. 1A and B). We also examined conidiation and found that, after seven days on SDC medium, conidia formation was significantly reduced. The $\Delta$ Moyvh1 mutant produced approximately $50 \%$ fewer conidia than Guy11 (Fig. 2A and B). This result indicated that MoYvh1 has a role in the regulation of both vegetative growth and conidia formation.

\section{MoYvh1 contributes to the pathogenicity of M. oryzae.}

To examine the involvement of MoYvh1 in pathogenicity, conidial suspensions of the $\Delta$ Moyvhl mutant, Guy11, and the complemented strain were sprayed onto susceptible rice seedlings (cv. CO-39) as described by Wang et al. (2013). After seven days, the $\Delta M o y v h l$ mutant caused disease lesions on the rice (Fig. $3 \mathrm{~A}$ and $\mathrm{B}$ ); however, the $\Delta M o y v h 1$ mutant failed to cause severe type 4 or 5 lesions and most of the lesions were type 1 and 2 and a very few were type 3 (Fig. 3C). On barley leaves inoculated with $5 \times 10^{4}$ spores per milliliter, the $\Delta M o y v h 1$ mutant caused less-severe lesions with restricted expansion of the yellow halo around the lesions (Fig. 4D).

\section{MoYvh1 is required for appressorium turgor pressure and invasive hyphae growth.}

To further understand the mechanism underlying reduced pathogenicity of the $\Delta$ Moyvhl mutant, we first examined appressorium formation on the $\Delta M o y v h l$ mutant. and there is no significant difference with the wild type (Supplementary Table S1). The appressoria turgor of the $\Delta$ Moyvhl mutants showed decreased collapse rates in the presence of 1,2 , and $3 \mathrm{M}$ glycerol (Fig. 4A), which was measured by a method described previously (Zhang et al. 2009b). We also examined the invasive hyphal growth on barley leaves and rice leaf sheaths 48 hpi with conidia suspensions based on the following four types: type 1, no penetration; type 2 , with penetration peg; type 3 , with a single invasive hypha; type 4 , with extensive hyphal growth (Chen et al. 2014b; Qi et al. 2016). In Guy11 and the complemented strains, more than $60 \%$ of cells showed type 4 $(73.96 \% \pm 1.31 \%$ and $77.43 \% \pm 1.74 \%)$ hyphae and only 20 to $25 \%$ were type $1(4.66 \% \pm 0.14 \%$ and $3.93 \% \pm 0.03 \%)$, type 2 $(8.36 \% \pm 0.23 \%$ and $5.93 \% \pm 0.41 \%)$, or type $3(13.02 \% \pm$ $0.83 \%$ and $12.71 \% \pm 0.77 \%$ ) hyphae. In contrast, less than $5 \%$ 
of the $\Delta$ Moyvhl cells were type $4(2.92 \% \pm 0.14 \%)$ hyphae and the rates of type $1(12.11 \% \pm 0.13 \%)$, type $2(58.73 \% \pm 1.84 \%)$, and type $3(26.24 \% \pm 0.97 \%)$ hyphae were greater than $90 \%$ (Fig. 4B and C). Similar results were observed in infected rice leaf sheaths. After incubation with spore suspensions for $48 \mathrm{~h}$, most invasive $\Delta$ Moyvhl hyphae were restricted to the primary infected rice leaf sheath cells, while invasive hyphae spread freely in the Guy11 and complemented strains (Fig. 4D). These results suggested that MoYvh1 plays an important role in turgor pressure and infectious hyphal growth.

\section{MoYvh1 is involved in the transfer}

\section{of the intracellular glycogen.}

M. oryzae conidia contain several storage carbohydrates, including mannitol, trehalose, and glycogen (Dixon et al. 1999). Since MoYvh1 was required for appressorium turgor, we suspected that the cellular distribution of glycogen during appressorium formation was altered in the $\Delta M o y v h 1$ mutant. Conidia from
A

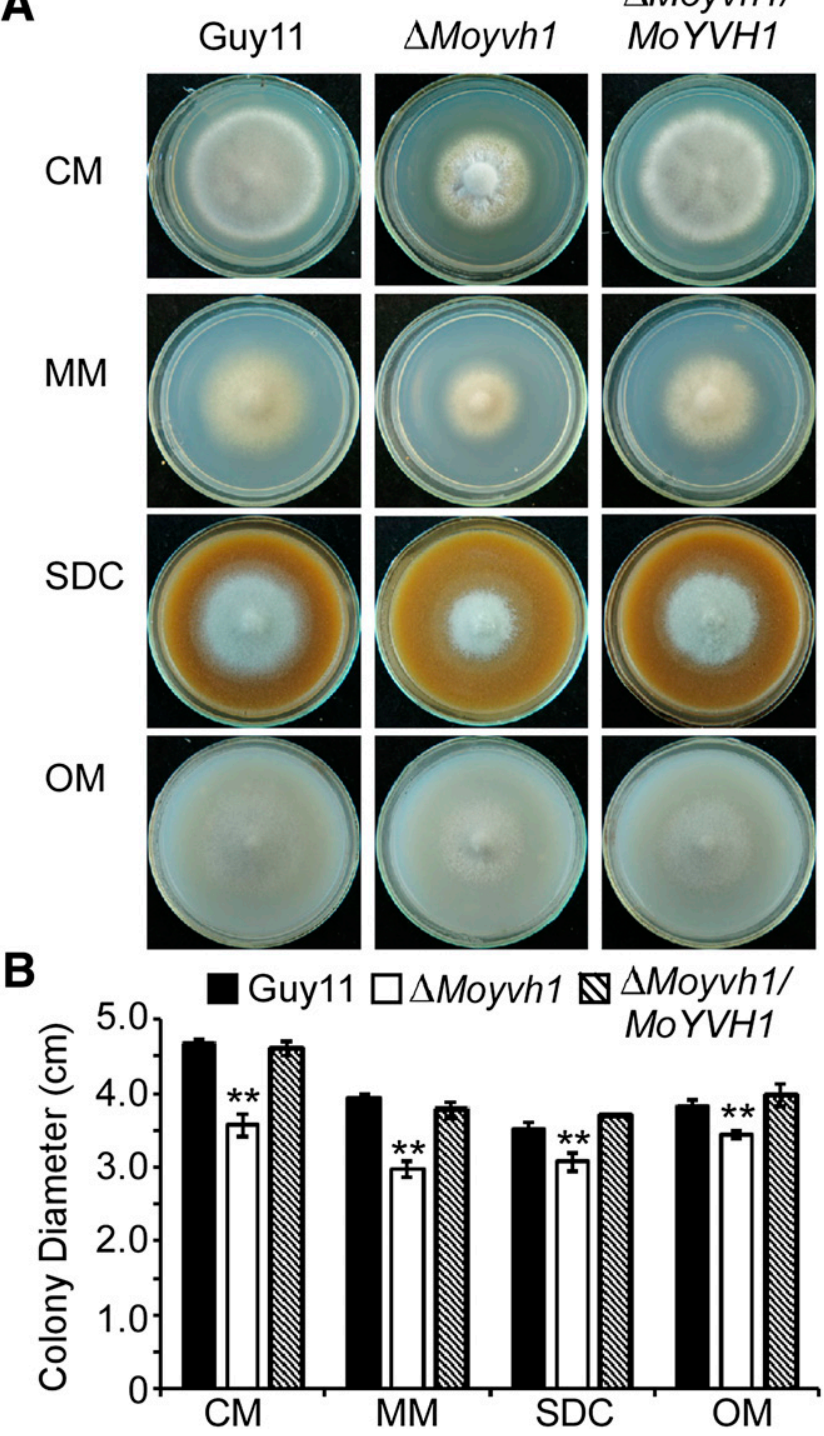

Guy11, the $\Delta$ Moyvhl mutant strains, and the complemented strains were allowed to germinate in water and to form appressoria on hydrophobic plastic cover slips (Supplementary Fig. S5). At various time points, they were incubated in a glycogen staining solution (60 $\mathrm{mg} \mathrm{KI}$ and $10 \mathrm{mg} \mathrm{I}_{2}$ ), according to Weber et al. (1998). Brown glycogen deposits became visible immediately. In the first $4 \mathrm{~h}$ prior to the formation of nascent appressoria, all samples showed an identical phenotype, but the glycogen contents of the topmost cells of conidia of Guy11 and the complemented strain reduced significantly. In contrast, the conidia of the $\Delta$ Moyvhl mutant contained high levels of glycogen after $2 \mathrm{~h}$. Over the next $12 \mathrm{~h}$, glycogen was transferred gradually into mature appressoria; at $20 \mathrm{~h}$, most $(71.33 \% \pm 5.77 \%)$ conidia in $\Delta$ Moyvhl mutant were still stained by the solution. Nevertheless, the Guy11 and complemented strain showed few conidia $(3.96 \% \pm 0.83 \%)$. These results indicated that the deletion of MoYVH1 disrupted transfer of the intracellular glycogen.

MoYvh1 has a role in mediating oxidative stress and scavenging ROS generated by the host plant.

Phosphorylation can be greatly enhanced by treatment with the inhibitor sodium orthovanadate or peroxy derivatives (Gordon 1991; Posner et al. 1994). To confirm the involvement of MoYvh1 in the oxidative stress response, we treated Guy11, the $\Delta$ Moyvhl mutants, and the complemented strains with various oxidative stress reagents, including butanediamide, Rose Bengal sodium salt, potassium peroxide $\left(\mathrm{KO}_{2}\right)$, and hydrogen peroxide $\left(\mathrm{H}_{2} \mathrm{O}_{2}\right)$. Mycelial growth of the $\Delta M o y v h l$ mutant was reduced significantly compared with Guy 11 . Moreover, $\Delta M o y v h l$ did not grow in the presence of high concentrations of oxidative stressinducing agents (Supplementary Fig. S6A and B).

ROS accumulation functions as a secondary signal that mediates plant defense (Alvarez et al. 1998; Gechev and Hille 2005; Lamb and Dixon 1997; Nurnberger et al. 2004), and the activation of ROS scavenging by phytopathogens is a mechanism for coping with plant defenses to facilitate infection (Chi et al. 2009; Guo et al. 2010). We examined host-derived ROS levels by staining with 3,3'-diaminobenzidine (DAB) at $24 \mathrm{hpi}$. Rice cells with Guy11 infectious hyphae were not stained by DAB and no reddish-brown precipitate was observed in infected cells. The primary infected rice cells with infectious hyphae of the $\Delta M o y v h 1$ mutant were stained intensely by $\mathrm{DAB}$, with reddish-brown precipitate around the appressoria and infected cells, indicating that the $\Delta$ Moyvhl mutant failed to scavenge accumulated $\mathrm{H}_{2} \mathrm{O}_{2}$ at the site of infection (Fig. 5A).
A

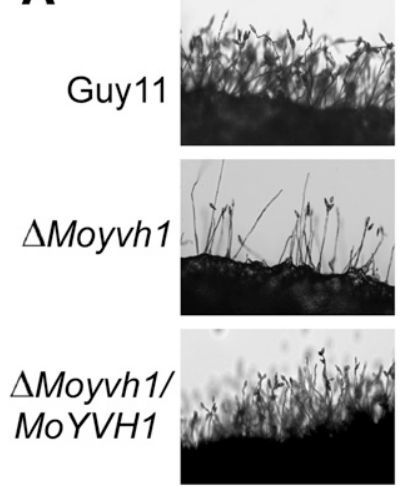

B Guy11 $\square \Delta$ Moyvh1

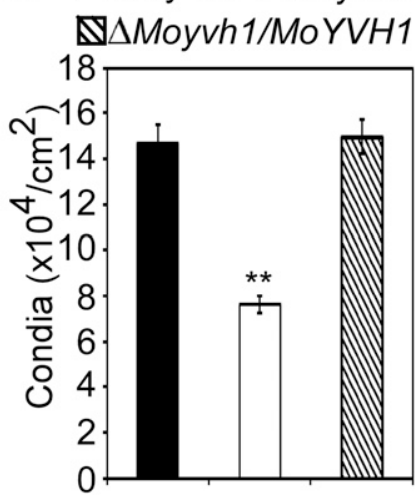

Fig. 1. MoYvh1 is required for normal mycelial growth on various medium. A, Comparison of $\Delta$ Moyvhl and wild-type strain in colony morphology. Wild type, the $\Delta M o y v h 1$ mutant, and the complementation strain were cultured at $28^{\circ} \mathrm{C}$ in darkness for 7 days. B, Statistical analyses of the diameter of hypha of wild-type Guy11, the $\Delta$ Moyvh1 mutant, and the complemented strain on different medium. Error bars represent the standard deviations; asterisks denote statistical significances $(P<0.01)$.
Fig. 2. The $\Delta$ Moyvhl deletion mutant showed reduced conidiation. A, The conidia were developed on straw decoction and corn agar (SDC) medium for 7 days and were examined by light microscopy. B, Statistical analysis of conidia production. The conidia produced by the wild type, mutant, and complemented strain grown on SDC medium for 10 days were collected, counted, and analyzed; asterisks denote statistical significances $(P<0.01)$. 
We assumed that the decreased rate of hyphal growth in infected cells and reduced virulence of the $\Delta M o y v h 1$ mutant might be due to a lack of ROS scavenger production by the host plant. To evaluate this hypothesis, an excised leaf-sheath assay was performed using diphenyleneiodonium (DPI) to inhibit the activity of plant NADPH oxidases, which are important generators of ROS (Bolwell et al. 1998; Grant et al. 2000; Jones and Dangl 2006; Zhang et al. 2009a). After incubation at $28^{\circ} \mathrm{C}$ for $36 \mathrm{~h}$, cells were observed under a light microscope. Without DPI treatment, the $\Delta$ Moyvhl mutant infectious hyphae in plant cells appeared swollen and were restricted to primary infected cells, while those of Guy11 spread into adjacent cells. Upon treatment with $0.5 \mu \mathrm{M}$ DPI to inhibit ROS generation, infectious hyphae of the $\Delta$ Moyvhl mutant spread into neighboring cells. In $M$. oryzae, conidia suspension carried elicitor activity that induced the production of $\mathrm{H}_{2} \mathrm{O}_{2}$ in rice cells that was dependent on fungal isolates and catalase activity in the suspension (Tanabe et al. 2009). Conidia suspensions harvested from Guy11, the $\Delta$ Moyvhl mutants, and the complemented strains were mixed with catalase of Aspergillus niger (CAG, Sigma) and were inoculated on the leaf sheath to control $\mathrm{H}_{2} \mathrm{O}_{2}$ around the infection site. After $36 \mathrm{~h}$, the defects of infection in $\Delta$ Moyvhl mutants appeared suppressed (Fig. 5A). This observation suggested that scavenging of host-derived ROS at the infection site was important for virulence of the $\Delta$ Moyvhl mutant and that deletion of $M o Y V H 1$ might explain the absence of infectious growth.

Therefore, we determined whether the $\Delta M o y v h 1$ strains elicited stronger defense responses than Guy11 during infection of rice. Transcript levels of pathogenesis-related (PR) genes were higher in rice plants infected with the $\triangle M o y v h 1$ mutants than with Guy11. PRIa, PR4, PBZ1, PAL1, and LOX1 expression levels at 24 hpi during infection by the $\triangle M o y v h l$ strains were higher than during infection by Guy11 as determined by realtime qPCR analysis (Fig. 5C). At 24 hpi, Guy11 had already prevented the accumulated $\mathrm{H}_{2} \mathrm{O}_{2}$ at the infection site. Moreover, Guy11 was spreading into adjacent cells at this time. Based on these results, we concluded that restriction of the $\Delta$ Moyvh1 mutant in rice cells might be due to host-derived ROS at the infection site and the subsequent activation of strong plant defense responses.

\section{MoYvh1 reduces the activities of extracellular peroxidases and laccases.}

$\mathrm{CR}$ binds to the cell-wall component $\beta$-1,4-glucan and is often used to assess CWI (Chi et al. 2009; Wood and Fulcher 1983). CR can also be used to detect secreted peroxidases, because it can be hydrolyzed by fungal peroxidases (Chi et al. 2009; Zhang et al. 2011b). To investigate whether MoYvh1 plays a role in CWI, CR $(200 \mu \mathrm{g} / \mathrm{ml})$ was added to CM medium. Inhibition of mycelial growth was significantly higher in the $\Delta$ Moyvhl mutant $(17 \%)$ than in Guy11 $(8 \%)$, and the golden halo (which indicates CR degradation) was smaller around the $\Delta$ Moyvh1 mutant than around Guy11 (Supplementary Fig. S7A). These results indicated that the Moyvhl mutant lost the peroxidase activity. Similarly, the extracellular laccase activity was also reduced in the $\Delta M o y v h 1$ mutant compared with Guy11. We hypothesized that the decreased extracellular peroxidase and laccase activity in the $\Delta M o y v h l$ mutant might be due to reduced transcription of genes encoding these enzymes, so we then examined the expression of several extracellular peroxidase and laccase genes in the $\Delta$ Moyvhl mutant, by qRT-PCR. We found that the transcript levels of six peroxidase-encoding genes but not MGG_11856 and all nine genes encoding putative laccases (Guo et al. 2010) were significantly reduced in the $\Delta$ Moyvhl mutant. These results suggested that deletion of $M o Y V H 1$ resulted in reduced peroxidase and laccase activities.
MoYvh1 disruption suppresses conidia formation and pathogenicity by reducing cAMP levels.

Overexpression of $S c P D E 2$ in the $\triangle S c y v h 1$ mutant could suppress the defects caused by deletion of $S c Y V H 1$ in $S$. cerevisiae (Beeser and Cooper 2000). To examine whether MoYvh1 is also linked to cAMP signaling, we measured intracellular cAMP levels in mycelia of the $\Delta$ Moyvhl mutant and found that the $\Delta$ Moyvhl mutant exhibited a 1.5 -fold higher intracellular cAMP level than the wild-type Guy11 and the complemented strain (Fig. 6A). Examination of the MoPDEH expression level also showed decreased expression in the $\Delta$ Moyvh1 mutant (approximately 0.5 -fold). We further overexpressed $M o P D E H$ in the $\triangle M o y v h 1$ mutant to determine if MoPdeH could restore defects caused by MoYVH1 deletion.

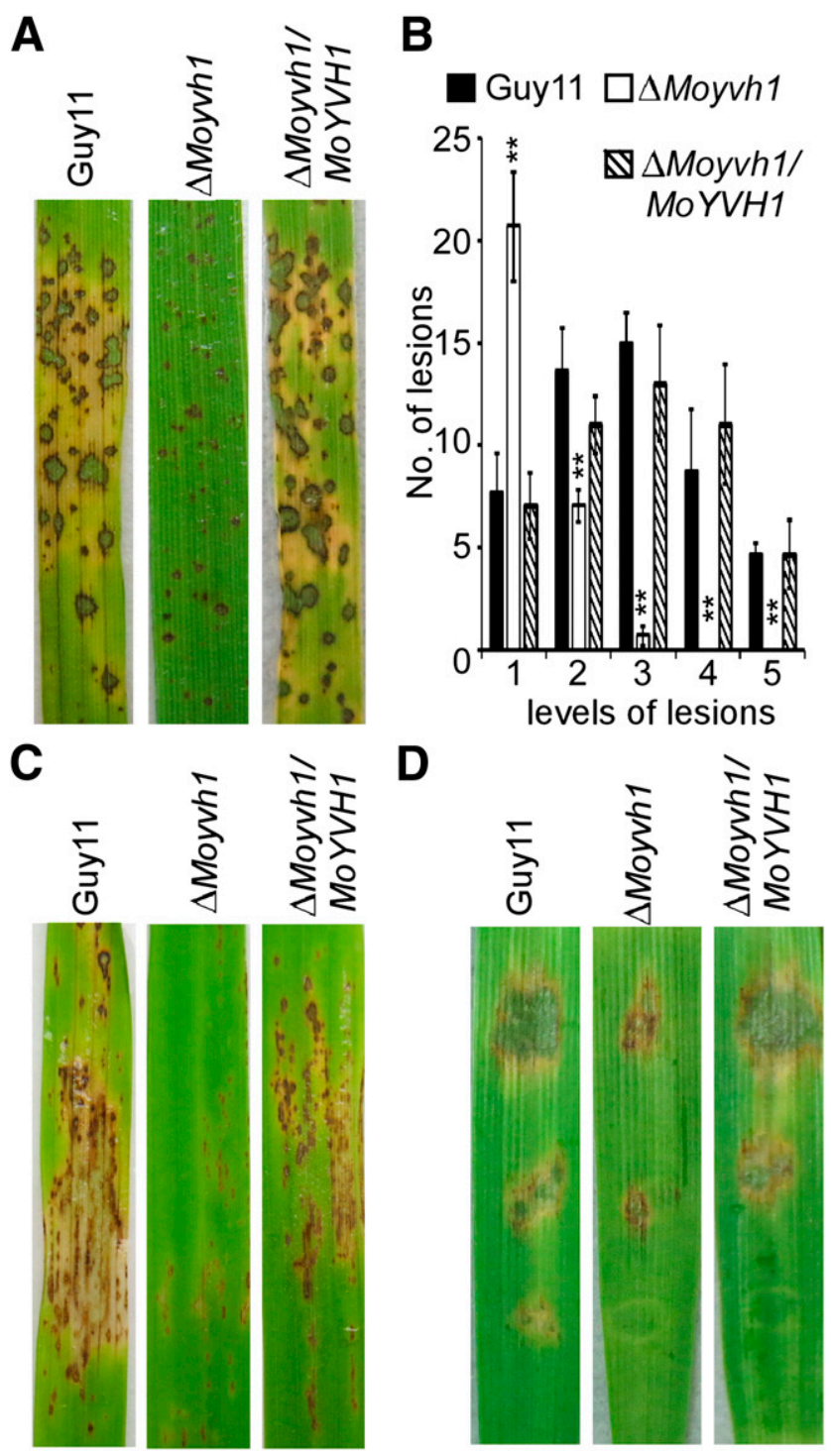

Fig. 3. MoYVH1 deletion decreased pathogenicity. A, The pathogenicity on rice leaves. B, Quantification of lesion type $(0=$ no lesion, $1=$ pinheadsized brown specks, $2=1.5-\mathrm{mm}$ brown spots, $3=2$ - to 3 - $\mathrm{mm}$ gray spots with brown margins, $4=$ many elliptical gray spots longer than $3 \mathrm{~mm}$, $5=$ coalesced lesions infecting $50 \%$ or more of the leaf area). Lesions were photographed and were measured at 7 days postinoculation, numbers within an area of $4 \mathrm{~cm}^{2}$ were counted, and experiments were repeated three times with similar results. Asterisks indicate significant differences at $P=0.01$. C, Leaves from 4-week-old rice seedlings were injected with conidial suspension $\left(5 \times 10^{4}\right.$ spores/ml $)$ of Guy11, the DMoyvhl mutant, and the complemented strain. Diseased leaves were photographed 7 days after inoculation. D, Pathogenicity test on barley leaves. 
Several transformants were obtained and tested by qRT (Fig. 6B). One of these, $\triangle M o y v h 1 / o x M o P D E H-5$, in which the $M o P D E H$ expression level increased to $160 \%$ that in the wild type, was able to decrease intracellular cAMP levels (Fig. 6A) and suppress the defects in conidia formation and pathogenicity, although partially. However, the transformant $\triangle M o y v h 1 / o x M o P D E H-3$, in which $M o P D E H$ expression level was almost the same as that of the $\triangle$ Moyvhl mutant, could not complement the cAMP levels and defects (Fig. 6C and D). Overexpressed MoPdeH suppressed the defects in sporulation and pathogenicity of $\mathrm{MoYVHI}$ deletion, the growth defect was not restored in both $\triangle M o y v h 1 / o x M o P D E H-3$ and $\triangle M o y v h 1 / o x M o P D E H-5$ strains (Supplementary Fig. S8A). Since higher intracellular cAMP levels and defects in conidia formation and pathogenicity were attributed to low levels of $M o P D E H$ expression in the $\triangle M o y v h l$ mutant, these results were consistent with that of MoYvh1 involved in the regulation of intracellular cAMP levels mediated by MoPdeH.

\section{MoYVH1 is required for the maintenance \\ of CWI and the regulation of the MAPK pathway.}

Recently, we found that MoPdeH functions upstream of the MoMck1-MoMkk1-MoMps1 MAPK pathway to regulate CWI (Yin et al. 2015). We overexpressed the MoMCKl in the $\triangle M o y v h l$ mutant (Supplementary Fig. S9) and found that it could suppress the defects in conidiation and virulence caused by MoYVHI deletion (Fig. 6C and D). To test whether MoYvh1 functions upstream of MoPdeH to regulate this MAPK pathway, we measured mycelial growth on CM medium containing various concentrations of CR. After cultured for 7 days, the $\Delta M o y v h 1$ mutant was significantly more sensitive to all CR concentrations tested (Fig. 7C). However, the $\triangle M o y v h 1 / o x M o M C K 1$ transformants showed more tolerance than the $\Delta$ Moyvhl mutant.

We then tested the sensitivity of the strains to cell walldegrading enzymes. Equal amounts of mycelia from the $\Delta$ Moyvh1 mutant, Guy11, and the complemented strain were treated with cell wall-degrading enzymes. After incubation at $30^{\circ} \mathrm{C}$, the $\Delta$ Moyvhl mutants were more readily digested and released more protoplasts than the Guy 11 and complemented strain (Fig. 7A and B). We further quantified the chitin contents of these cell types and found that the chitin content of the $\Delta$ Moyvh1 mutant was reduced by $50 \%$ compared with Guy 11 (Fig. 7D). These results indicated that MoYvh1 is required for maintenance of CWI.

The MAPK cascade is thought to act upstream of CWI signaling in S. cerevisiae. In M. oryzae, the MoMck1 and MoMps1 proteins, which participate in MAPK signaling, have been reported to be crucial for CWI and pathogenicity. Therefore, we assayed Mps1 activation in the $\Delta M o y v h 1$ mutant and found that the phosphorylation of Mps1 increased in the mutant (Fig. 7E). Taken together, these results demonstrated that MoYvh1 functions in the maintenance of CWI via the MAPK pathway.

\section{Functional characterization of different domains of MoYvh1.}

MoYvh1 has conserved N-terminal phosphatase and Cterminal zinc-binding domains. To assess whether the phosphatase domain or the zinc-binding domain is important for the growth, conidiation, and pathogenicity of MoYvh1, several plasmids harboring $\mathrm{MoYVHl}$ fused to a green fluorescent protein (GFP) coding sequence were constructed, including MoYvh1 ${ }^{\Delta \mathrm{C}_{-}}$ GFP (deletion of the C-terminal region), MoYvh1 ${ }^{\Delta \mathrm{N}}$-GFP (deletion of the N-terminal region), and MoYvh1 ${ }^{\text {C123S }}$-GFP (point mutation of the active site). Expression of MoYvh1 ${ }^{\Delta \mathrm{N}_{-}}$GFP and MoYvh1 ${ }^{\mathrm{C} 123 \mathrm{~S}}$-GFP complemented the defects in the $\Delta$ Moyvhl strains. In contrast, MoYvh1 ${ }^{\Delta \mathrm{C}}-\mathrm{GFP}$ caused phenotypes similar to those of the $\Delta$ Moyvhl mutant in vegetative growth, conidia formation, and pathogenicity (Fig. 8). Further experiments in cAMP, oxidative stress, osmotic stress, the chitin content, protoplast release, DAB assay, and, also, the transfer of glycogen showed similar results that only C-termainal domain complement the defects in the $\Delta M o y v h 1$ mutant strains (Supplementary Fig. S11). Taken together, these findings suggest that the C-terminal zinc-binding domain is essential for the roles of MoYvh1.

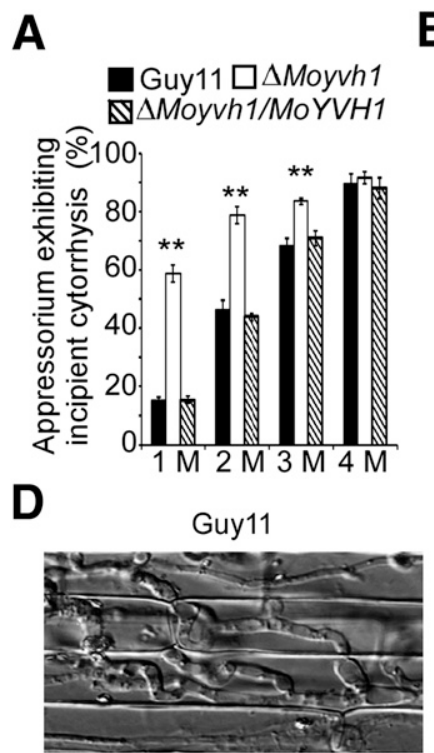

$88.33 \pm 5.81 \%$
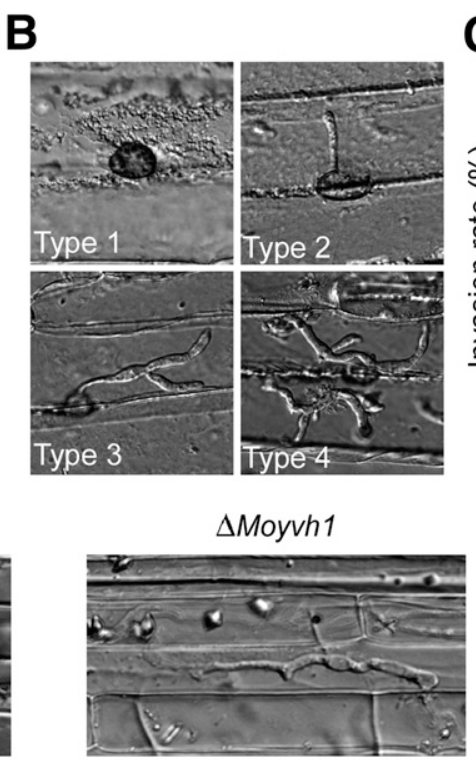

$73.73 \pm 3.86 \%$
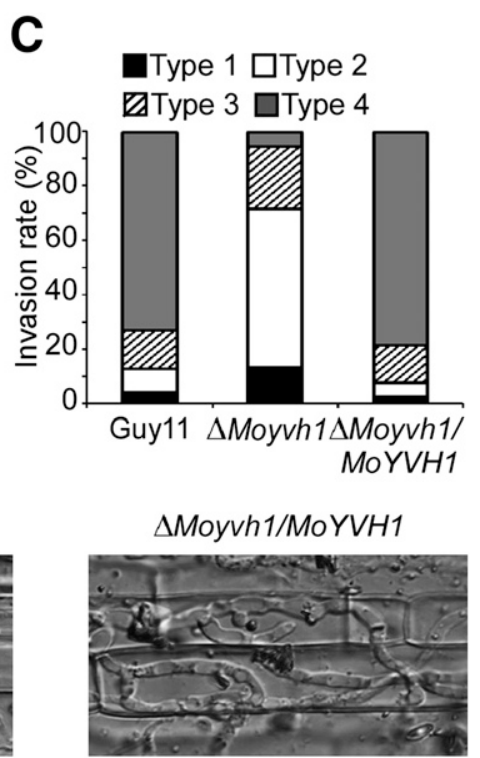

$83.16 \pm 5.27 \%$

Fig. 4. MoYVH1 deletion decreased the infection on barley and rice. A, Different concentrations of glycerol (1 to $4 \mathrm{M})$ were used for cytorrhysis analysis. For each concentration, at least 100 appressoria were counted and the experiment was repeated three times with the same result; asterisks denote statistical significances $(P<0.01)$. B, Excised barley leaves from 7 -day-old barley seedlings were inoculated with conidial suspension $\left(1 \times 10^{5}\right.$ spores/ml $)$ Infectious growth was observed at $24 \mathrm{~h}$ postinoculation (hpi). C, Statistical analysis for each type of infectious hyphal shape. For each tested strain, 100 infecting hyphae were counted per replicate and the experiment was repeated three times. D, The conidial suspension $\left(1 \times 10^{5}\right.$ spores/ml $)$ was inoculated in excised rice sheath of 4-week-old rice seedlings. The invasive hyphae growth was observed at 48 hpi. 


\section{DISCUSSION}

In this study, we examined the conserved putative protein phosphatase MoYvh1 from M. oryzae, which is homologous to the S. cerevisiae YVH1 protein and participates in vegetative growth, conidia formation, pathogenicity, and the cAMP-dependent protein kinase cascade. Our data furthers understanding of the PKAmediated cAMP signaling pathway by showing that the regulator is involved in adjusting intracellular cAMP levels. Our findings provide important insights into the molecular mechanisms underlying the pathogenicity of rice blast fungus.

In plants, the generation of ROS is regarded as one of the first responses to fungal invasion (Mellersh et al. 2002). For plant pathogens, ROS also play an important role and loss of the ability to eliminate intracellular ROS can result in functional defects (Egan et al. 2007). In M. oryzae, $\Delta$ Moatfl mutant was absent in scavenging the ROS around invasion hyphae and inhibition of the plant NADPH oxidase by DPI restored infectious hyphal growth of the mutants in rice cells (Guo et al. 2010). Moreover, deletion of the bZIP transcription factor MoAPl that mediates the oxidative stress response showed accumulation of ROS at the infection site and lack of invasive hyphae growth (Guo et al. 2011). In this study, we found that the $\Delta M o y v h l$ mutants had no apparent defects in appressorium formation but showed restricted infectious hyphae growth and reduced virulence on the host (Fig. 4D). In view of the sensitivity of the mutant strain to $\mathrm{H}_{2} \mathrm{O}_{2}$, we suspected that such a result may be due to the loss of ROS-degrading ability in the mutants, and this hypothesis was substantiated by the DAB staining assay. Furthermore, after treatment with DPI and catalase, the defect in invasion was suppressed, which is agreed with MoAtf1 and MoAp1. Taken together with our findings on the induced expression of PR genes in rice inoculated with $\Delta$ Moyvhl mutants (Fig. 5C), these observations suggested that MoYvh1 participates in inhibition of the plant defense response. We concluded that MoYVH1 is involved in the disruption of extracellular ROS and avoiding host immune response is responsible for the virulence.

cAMP signaling plays an important role in regulating M. oryzae growth and differentiation (Ramanujam and Naqvi 2010), and our previous study demonstrated that deletion of $M o P D E H$ resulted in a high intracellular cAMP concentration and significantly reduced sporulation and pathogenicity (Zhang et al. 2011a). Independently, Ramanujam and colleagues (2010) harvested more conidia from a $\triangle M o p d e H$ mutant than from a wild-type strain (B157) and they suggested that a high cAMP level contributes to conidiation. MoRGS1, regarded as one of
A

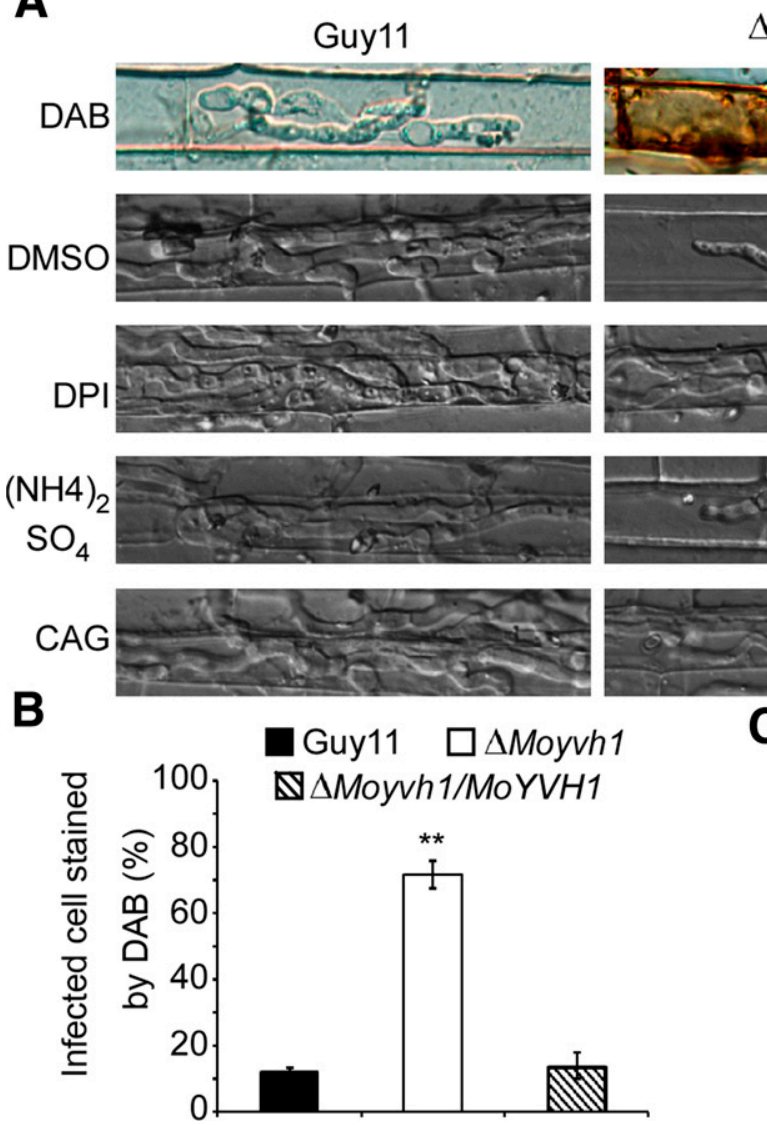

Moyvh1
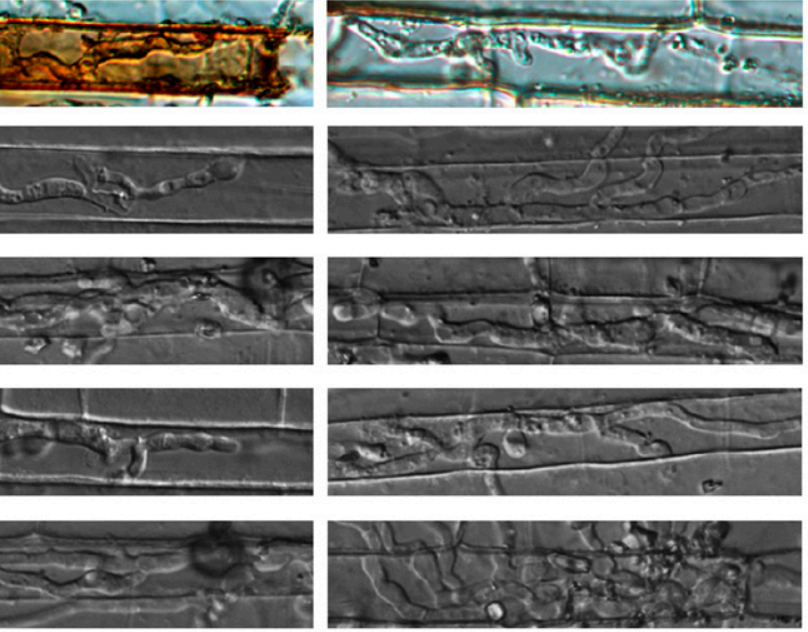

\section{C}

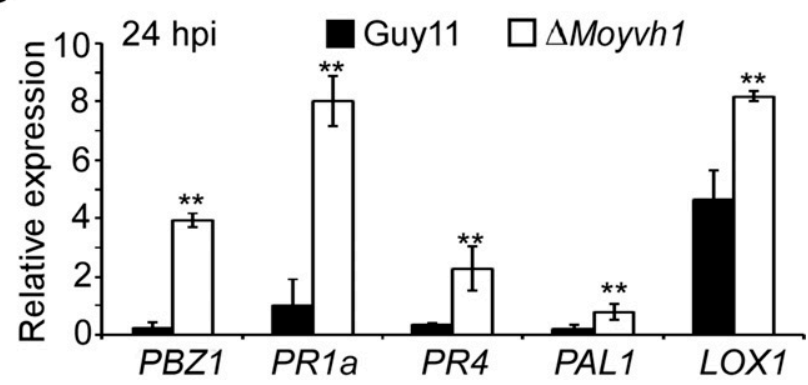

Fig. 5. The MoYVH1 deletion mutant induced strong plant defense responses. A, 3,3'-Diaminobenzidine (DAB) staining of the excised leaf sheath of rice infected by Guy11, the $\Delta M o y v h 1$ mutant, and the complemented strain $24 \mathrm{~h}$ after inoculation. The excised sheath of rice was inoculated with conidial suspension after treatment with or without $0.5 \mu \mathrm{M}$ diphenyleneiodonium (DPI) dissolved in dimethyl sulfoxide (DMSO) by three independent experiments. The rice sheath was also inoculated with conidial suspension after treatment with or without $0.2 \mathrm{U}$ catalase of Aspergillus niger (CAG, Sigma) dissolved in $10 \mathrm{mM}(\mathrm{NH} 4)_{2} \mathrm{SO}_{4}$. Samples were harvested and were observed $36 \mathrm{~h}$ after inoculation. This experiment was performed with three independent experiments and representative results from one of these experiments are presented. B, The infected cell stained by DAB. Three independent biological experiments were performed, with three replicates each time, yielding similar results in each independent biological experiment. Error bars represent standard deviation; asterisks represent significant difference between the different strains $(P<0.01)$. C, The transcription of PBZ1, PRIa, PR4, PAL1, and LOX1 in the infected rice was assayed using quantitative reverse transcription-polymerase chain reaction. RNA samples were collected from rice plants $24 \mathrm{~h}$ after inoculation with the $\Delta$ Moyvh1 mutant or wild-type strain. The average threshold cycle of triplicate reactions was normalized by the stable-expressions gene elongation factor $1 \alpha$ (Os03g08020) in Oryza sativa. Three independent biological experiments were performed and yielded similar results. Error bars in the figure represent standard deviation; asterisks denote statistical significances $(P<0.01)$. 
the major regulators of $\mathrm{G}$ protein signaling, also regulates cAMP levels in $M$. oryzae, and the $\Delta$ Morgs 1 mutant strains exhibit a higher cAMP level but reduced conidial production (Liu et al. 2007; Zhang et al. 2011b). In this study, we found that the $\Delta M o y v h 1$ mutants produced smaller and restricted lesions and that the cAMP levels of the $\Delta M o y v h 1$ mutants were markedly higher than that of Guy11, these results agreed with the deletion of MoPDEH or MoRGS1 in M. oryzae. Moreover, overexpressed $M o P D E H$ in the $\triangle M o y v h 1$ mutant resulted in recovery of conidiation, pathogenicity (Fig. 6C and D) and, also, the ability to scavenge the ROS, which indicated that MoYvh1 influences the downstream PKA-cAMP pathway and the cAMP pathway plays important roles in regulating conidiation and virulence in $M$. oryzae.

Although the overexpression of MoPdeH suppressed the defects in sporulation and pathogenicity of $\Delta$ Moyvhl, the growth defect was not restored. cAMP plays roles in conidiation and pathogenicity of $M$. oryzae, however, it showed no evidence in regulating vegetative growth. The $\triangle M o p d e H \mathrm{mu}-$ tants that lead to threefold higher levels cAMP compared with the wild type showed no significant difference in colony morphology or growth rate on a variety of mediums (Zhang et al. 2011a). In addition, the MoPdeH-cAMP signaling pathway showed cross-talks with the CWI, and all of the MoMck1MoMkk1-MoMps1 MAPK cascade components are involved in regulating intracellular cAMP levels; however, the vegetative growth of all these mutants showed no difference from the wild type (Jeon et al. 2008; Xu et al. 1998; Yin et al. 2015).
Moreover, MoGti1, which possesses a PKA phosphorylation site that regulates the MoCpkA- and MoPmk1-mediated cAMP pathway, exhibited a slightly larger colony diameter than the wild-type strain (Chen et al. 2014a). According to these results, we indicated that cAMP might not have a direct relationship with vegetative growth and, also, the defects in growth of $\Delta$ Moyvhl mutant strains might not account for the cAMP-PKA signaling pathway.

The CWI signaling pathway allows $M$. oryzae to sense and respond to cell-wall stresses that arise during growth, differentiation, and pathogenicity (Jeon et al. 2008; Xu et al. 1998). In our study, the $\Delta M o y v h l$ mutant was sensitive to the CWI inhibitor CR and the phosphorylation ability of Mps1 of $\Delta M o y v h 1$ decreased, and also, the $\triangle M o y v h 1 / o x M o M C K 1$ transformants suppressed the defects on the CWI of the $\Delta M o y v h l$ mutant. In the $\triangle M o m c k 1$ mutant, the intracellular cAMP level increased and the expression of $M o P D E H$ was down-regulated, which suggests a feedback regulatory mechanism of MoPdeH that cross-talks between cAMP signaling and the MAPK cascade (Yin et al. 2015). Here, we showed that the intracellular cAMP level in the $\triangle M o y v h 1$ mutant increased, which probably resulted from the downregulated expression of MoPDEH (Fig. 6A and B). This type of regulation suggests that MoYvh1 functions upstream of MoPdeH-mediated cAMP-signaling and MoMck1-mediated CWI to affect asexual development and pathogenicity (Fig. 9).

In $S$. cerevisiae, the reduced growth rate of the $\Delta S c y v h 1$ mutants was completely restored in the $\triangle S c y v h 1 / S c M R T 4^{G 69 D}$ strains (Kemmler et al. 2009; Lo et al. 2009; Sugiyama et al.
A

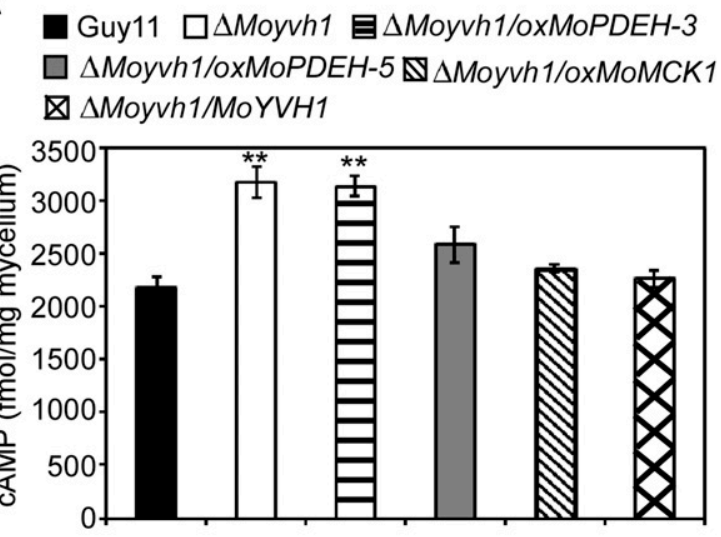

C

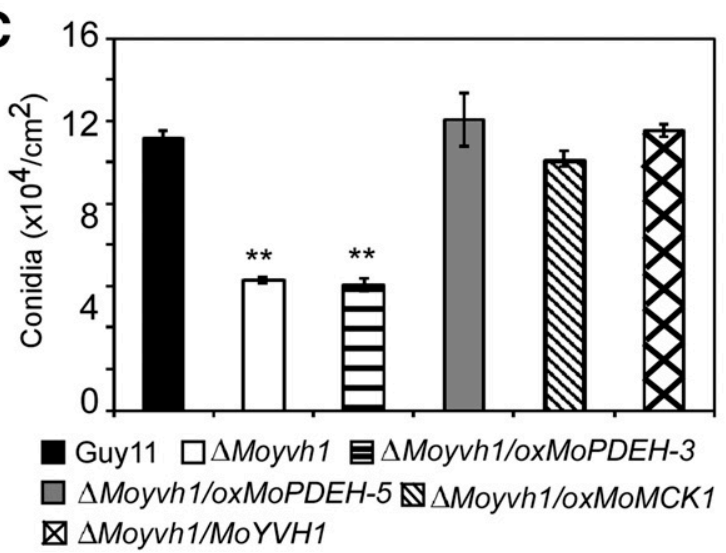

B

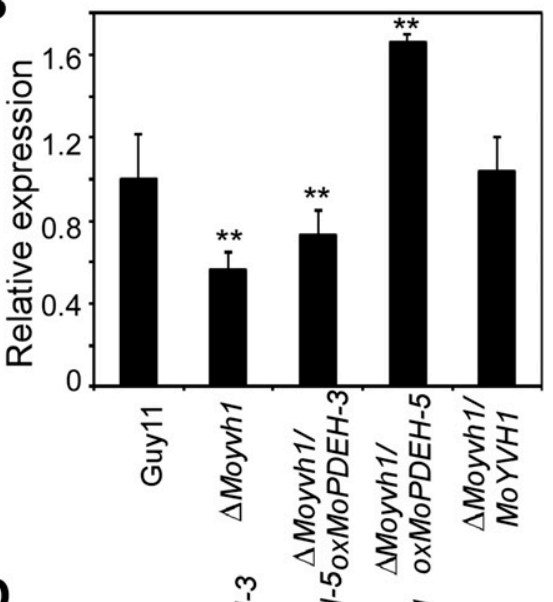

D

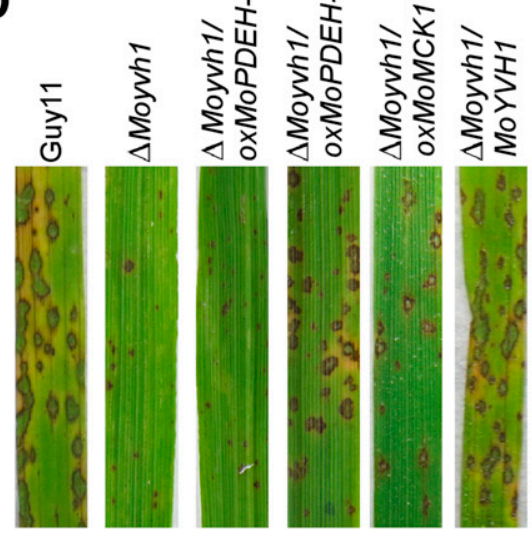

Fig. 6. MoYvh1 is involved in the cAMP signal pathway and is located upstream to MoPdeH. A, Quantification of intracellular cAMP in the mycelia stage of all the strains. The error bars represent standard deviation (SD) of three replicates. B, Expression analysis of the MoPDE2 gene in wild type and mutant strains. The error bars indicate SD of three replicates; asterisks indicate significant difference $(P<0.01)$. C, Conidia formation assay. Each strain was cultured in straw decoction and corn agar medium in the dark for 7 days; asterisks indicate significant difference $(P<0.01)$. D, Spraying assay. Conidial suspensions of each strain were sprayed on rice seedlings. Diseased leaves were photographed 7 days after inoculation. 
2011). ScMrt4 is thought to be involved in the early stages of ribosome synthesis, and signal transduction is affected by delayed ribosomal synthesis, due to damage to the protein splicing and processing machinery (Harnpicharnchai et al. 2001; Kemmler et al. 2009; Lo et al. 2009; Zuk et al. 1999). These data showed that ScYvh1-mediated release of ScMrt4 precedes cytoplasmic loading of ScRpp0 on pre-60S particles and the assembly of ribosome stalk requires the ScYvh1 for the exchange of ScMrt4 with ScRpp0 in S.cerevisiae (Kemmler et al. 2009; Lo et al. 2009). Therefore, we speculate that MoYvh1 might participate in the early stage of ribosomal synthesis that subsequently influences vegetative growth in $M$. oryzae; further studies focusing on this hypothesis are warranted.

MoYvh1 is a modular two-domain protein that contains a conserved phosphatase domain and a C-terminal zinc-binding domain, and our results indicated that MoYvh1 function is independent of the phosphatase domain but the C-terminal zinc-binding domain can completely complement the defects caused by the $\Delta$ Moyvhl mutation, including those affecting growth, conidia formation, pathogenicity, infection, and CWI (Fig. 8). In S. cerevisiae, inactivation of ScYvh1 phosphatase activity (Yvh1-C117A) or deletion of the entire N-terminal phosphatase domain did not cause a slow-growth phenotype (Beeser and Cooper 2000; Muda et al. 1999). Furthermore, mutations in which the entire $\mathrm{C}$-terminal zinc-binding domain was deleted were found to be responsible for the slow growth of $\Delta$ Scyvhl strains (Beeser and Cooper 1999; Liu and Chang 2009; Sakumoto et al. 1999), which is agreed with our results.
VH1 phosphatase was shown to have activity against tyrosineand serine-phosphorylated substrates; mutagenesis of an essential cysteine in the vaccinia phosphatase abolishes catalytic activity directed toward both substrates (Guan et al. 1992). Although the levels of phosphatase activity are very low, Muda et al. (1999) observed $\mathrm{ScYVHI}$ that have activity against several tyrosine-phosphorylated substrates. While no evidence has established how the N-terminal phosphatase domain of MoYvh1 functions in $M$. oryzae, we speculate that $M o Y V H 1$ might have a more restricted activity toward specific proteins under certain conditions in vivo and that the phenotype we have examined may be not reflective of the "true" function of the phosphatase domain. Since the C-terminal region of MoYvh1 complemented the defects, we assume that the zinc-binding domain is largely dispensable for the intrinsic enzymatic activity and its putative interaction with the endogenous target is essential for the vivo function. Roles for the zinc-binding domain might include correct localization of the catalytic core within cellular compartments or is involved in protein-protein interactions.

Our results revealed that the putative protein phosphatase MoYvh1 plays a critical role in M. oryzae. MoYvh1 is a multifunctional protein required for vegetative growth, conidiogenesis, the CWI pathway, and pathogenicity. We confirmed that MoYvh1 functions upstream of the cAMP-PKA signaling pathway, and further studies investigating other signaling pathways influenced by MoYvh1 and its interacting proteins would help to further explore the importance of protein phosphorylation in growth and pathogenicity of $M$. oryzae.
A

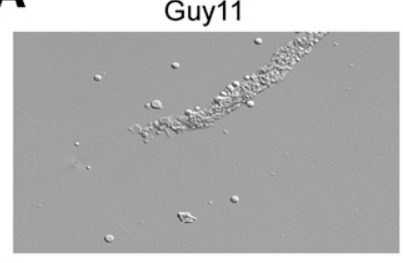

B

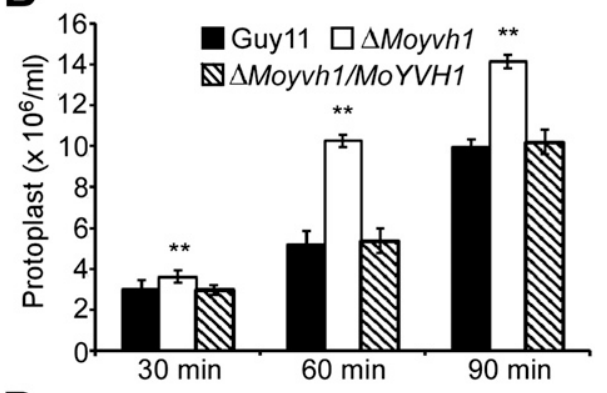

D

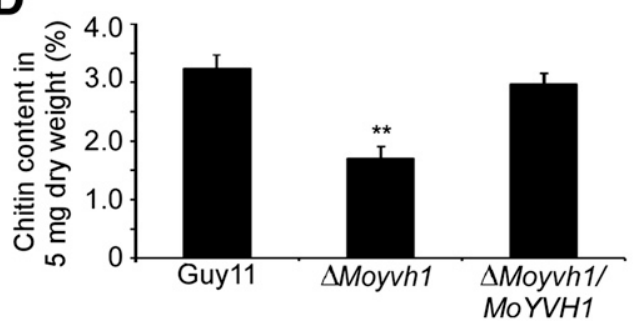

$\Delta$ Moyvh1

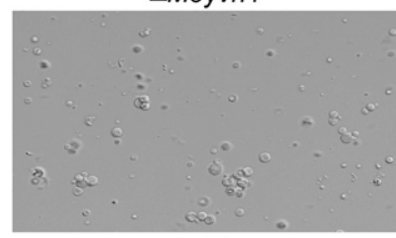

C

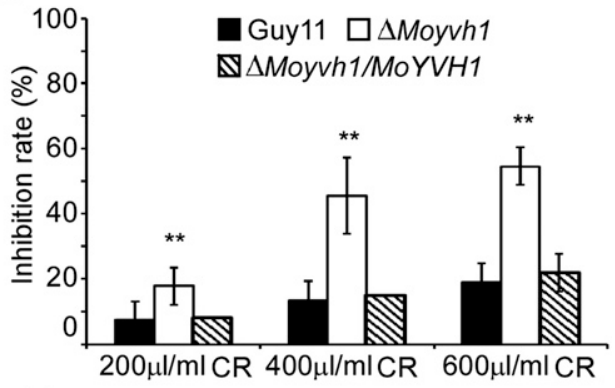

E

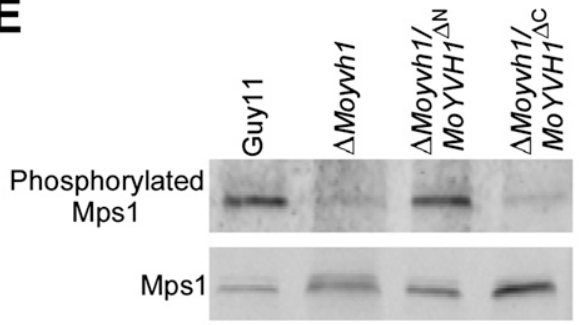

Fig. 7. MoYvh1 has a role in cell-wall integrity. A, Light microscopic examination of protoplasts release after treatment for 60 min and photographed. B, Protoplast production of Guy11, the $\Delta M o y v h 1$ mutant, and the complemented strain treated by cell-wall degrading enzyme. Asterisks indicate a significant difference between the mutant and wild-type strain at $P<0.01$. C, Wild-type Guy11, $\Delta M o y v h 1$ mutant, and the complemented strain were incubated on complete medium (CM) plates containing different concentrations of Congo Red $(\mathrm{CR})$ at $28^{\circ} \mathrm{C}$ for 7 days. The growth rate was determined by plotting the percentage of colonies in the presence of various concentrations of CR against regular CM; asterisks denote statistical significances $(P<0.01)$. D, GlcNa determination shows significantly decreased chitin contents in the $\Delta M o y v h 1$ mutant. Data comprise three independent experiments with triple replications each time that yielded similar results. Asterisks indicate significant differences at $P<0.01$. E, Proteins were prepared from Guy $11, \Delta M o y v h 1, \Delta M o y v h 1 / \mathrm{MoYvh1}{ }^{\Delta \mathrm{C}}$, and $\Delta M o y v h 1 / \mathrm{MoY} v \mathrm{~h} 1^{\Delta \mathrm{N}}$ mutant mycelia cultured in liquid CM media; signal intensity of phosphorylated Mps1 decreased in the $\Delta M o y v h 1$ and $\Delta M o y v h 1 / M o Y v h{ }^{\Delta C}$ mutant. 


\section{MATERIALS AND METHODS}

\section{Strains and culture condition.}

M. oryzae Guy11 strain was used as the wild type in this study. All strains were cultured on CM agar plates for 3 to 15 days at $28^{\circ} \mathrm{C}$ (Talbot et al. 1993). Fungal mycelia were harvested from liquid CM and were used for genomic DNA and RNA extractions. Protoplasts were prepared and transformed as described (Sweigard et al. 1992). Transformants were selected on TB3 medium (3 $\mathrm{g}$ of yeast extract, $3 \mathrm{~g}$ of casamino acids, $200 \mathrm{~g}$ of sucrose, and $7.5 \mathrm{~g}$ of agar in 1 liter of distilled water) with $300 \mu \mathrm{g}$ of hygromycin B per milliliter (Roche) or $200 \mathrm{~g}$ of zeocin per milliliter (Invitrogen).

qRT-PCR analysis.

For qRT-PCR, total RNA was reverse transcribed into firststrand cDNA using the oligo (dT) primer and M-MLV reverse transcription (Vazyme Biotech). qRT-PCR reactions were performed following previously established procedures and each experiment was repeated three times (Guo et al. 2010). qRTPCR was performed with the ABI 7500 Fast real-time system (ABI Co.) and transcripts were analyzed using the 7500 System SDS software (ABI Co.). To compare the relative abundances of target gene transcripts, the average threshold cycle $(\mathrm{Ct})$ was normalized to that of Actin (qRT-actinF/qRT-actinR) for each of the treated samples.

\section{MoYVH1 gene disruption complementation.}

To generate the MoYVH1 gene replacement vector pCX62, approximately $1-\mathrm{kb}$ upstream and $1-\mathrm{kb}$ downstream fragments were amplified with primer pairs (Supplementary Table S2). The resulting PCR products were ligated to the hygromycinresistance cassette released from pCX62, as previously described (Dong et al. 2015). Putative mutants were screened by

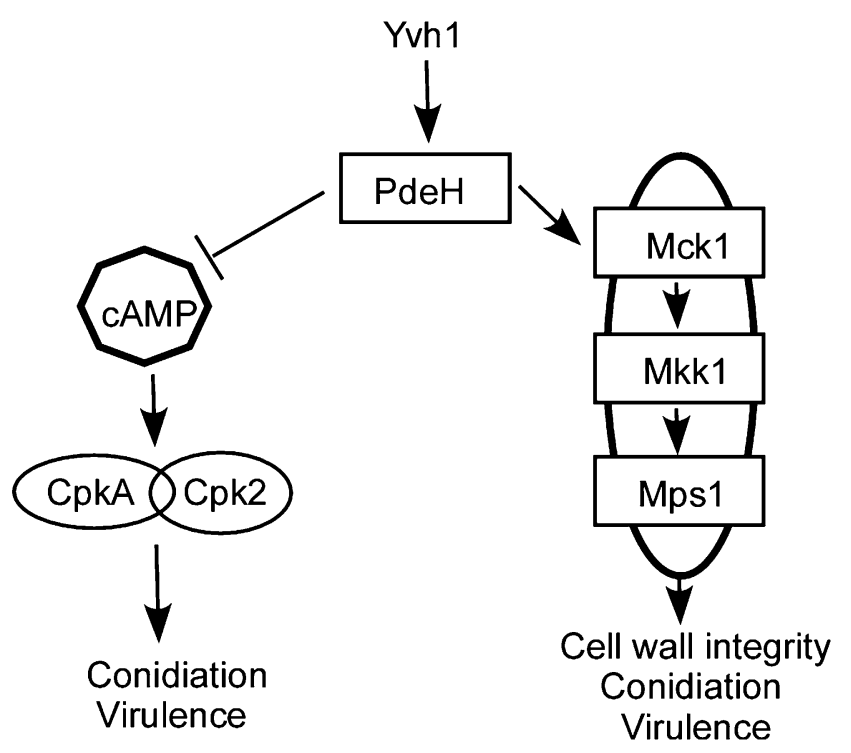

Fig. 9. A proposed model for the cross-talk between the cAMP pathway and the cell-wall integrety (CWI) pathway regulated by MoYvh1 in Magnaporthe oryzae. Putative cross-talks between the CWI pathway and cAMP pathway mediated by MoPdeH and regulated by the MoYvh1.

\section{Guy11}

$\Delta$ Moyvh1
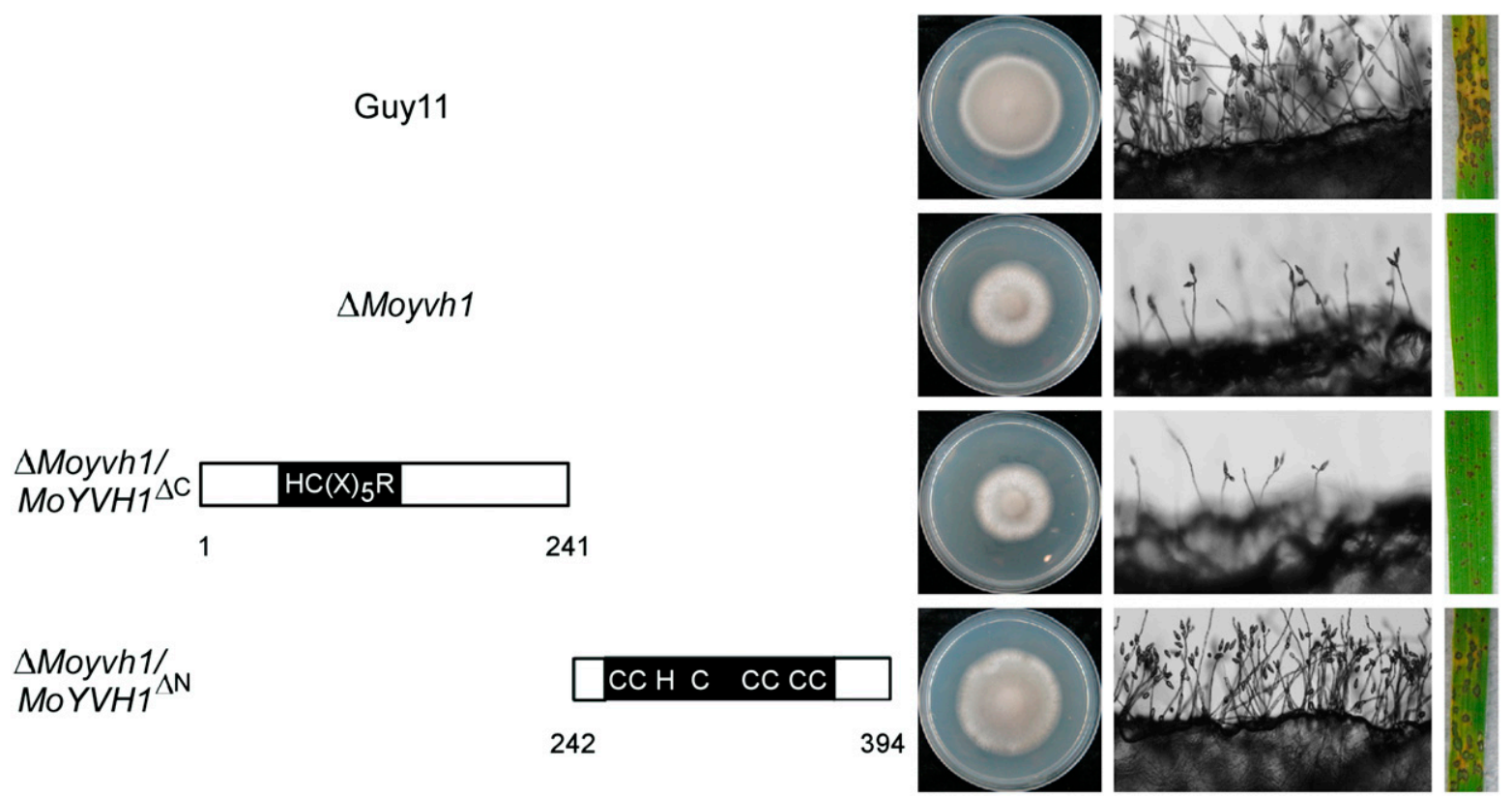

$$
\begin{aligned}
& \Delta \text { Moyvh1/ }^{\wedge \mathrm{N}} \\
& \text { MoYVH1 }^{\mathrm{N}}
\end{aligned}
$$

$\mathrm{CCHC \quad CCCC}$

242 $\mathrm{CCHC} \mathrm{CC} \mathrm{CC}$

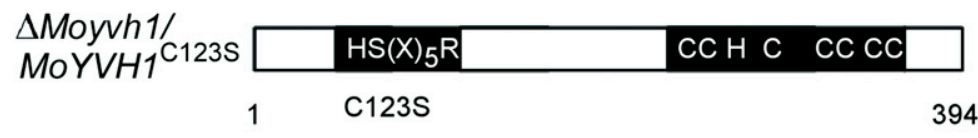

\section{$\triangle$ Moyvh1/
MoYVH1} $\mathrm{HC}(\mathrm{X})_{5} \mathrm{R}$

$\mathrm{CCHC} \quad \mathrm{CCCC}$
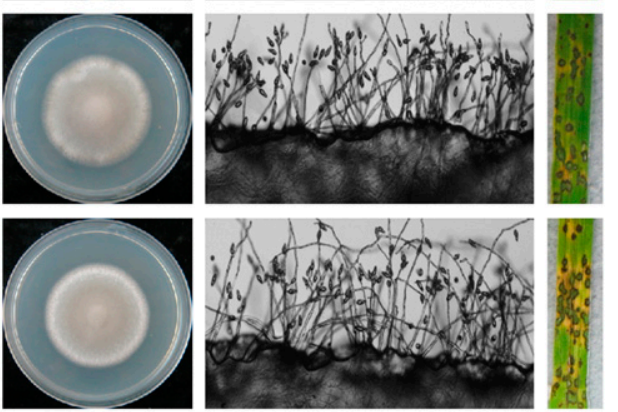

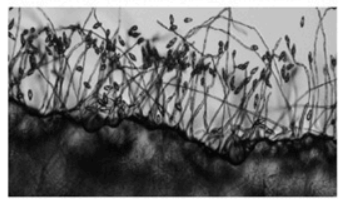

Fig. 8. Function of phosphatase domain and zinc-binding domain of MoYvh1. Vegetative growth, conidia formation, and pathogenicity in four different

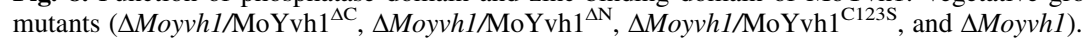


PCR and were confirmed by Southern blotting analysis. To complement the $\Delta M o y v h 1$ mutant, a DNA fragment including the putative promoter and the coding sequence was amplified and inserted into pYF11 (bleomycin resistance) by homologous recombination in Saccharomyces cerevisiae. The plasmids were extracted and were transformed into Escherichia coli-competent cells, and then, the plasmids with correct inserts were transferred into protoplasts, as previously described (Dong et al. 2015).

\section{Conidiation, appressorium formation, and turgor assays.}

After 7 days of cultivation, conidia were collected with $5 \mathrm{ml}$ of distilled water, were filtered through three layers of lens paper, and were counted with a hemacytometer under a microscope. Conidial germination and appressorium formation were measured on a hydrophobic surface (Qi et al. 2012). Conidial suspensions of $30 \mu \mathrm{l}\left(1 \times 10^{5}\right.$ spores per milliliter $)$ were dropped onto a hydrophobic surface and were placed in a moistened box at $28^{\circ} \mathrm{C}$. Appressorium formation rate was then calculated under the microscope at 12 hpi. More than 200 appressoria were counted for each strain and the experiment was repeated three times (Zhang et al. 2011a). Photographs were taken at $24 \mathrm{hpi}$. The appressorium turgor was measured using an incipient cytorrhysis (cell collapse) assay and a 1.0 to $4.0 \mathrm{M}$ glycerol solution (Zhang et al. 2009b). Conidial suspension the $\left(20 \mu \mathrm{l}, 5 \times 10^{4}\right.$ spores per milliliter $)$ were placed on plastic coverslips and were incubated for $24 \mathrm{~h}$ at $28^{\circ} \mathrm{C}$. The water surrounding the conidia was removed carefully and was replaced with an equal volume $(20 \mu \mathrm{l})$ of glycerol (1.0 to 4.0 $\mathrm{M})$. The number of appressoria that had collapsed after $10 \mathrm{~min}$ was recorded and at least 100 appressoria were counted for each strain. The experiments were repeated three times.

\section{Pathogenicity, plant infection, and rice-sheath penetration assays.}

Conidia were harvested from 10-day-old SDC agar cultures, were filtered through three layers of lens paper, and were resuspended to a concentration of $5 \times 10^{4}$ spores per milliliter in a $0.2 \%(\mathrm{wt} / \mathrm{vol})$ gelatin solution. For the leaf assay, leaves from two-week-old seedlings of rice (Oryza sativa cv. CO39) were used for spray inoculation and 7-day-old seedlings of barley were used for infection assays. Spray inoculation on the rice leaves, $5 \mathrm{ml}$ of a conidial suspension of each treatment were sprayed onto rice with a sprayer. Inoculated plants were kept in a growth chamber at $25^{\circ} \mathrm{C}$ with $90 \%$ humidity and in the dark for the first $24 \mathrm{~h}$, followed by a 12-h-light and 12-h-dark cycle. Lesion formation was observed daily and was photographed 7 days after inoculation (Guo et al. 2011).

\section{cAMP extraction and high-performance liquid chromatography (HPLC) analysis.}

All of the strains were cultured on CM medium at $28^{\circ} \mathrm{C}$, were cut into $1 \times 1$-mm squares, and were cultured in liquid $\mathrm{CM}$ for another 2 days. Filtering to collect mycelium and quickly ground into powder. Every $1 \mathrm{mg}$ of mycelium mixed with $20 \mu \mathrm{l}$ of $6 \%$ TCA solution was turbulenced. Samples were centrifuged $(1,377 \times g, 15 \mathrm{~min})$, the top layers were collected and were washed twice with five times the volume of anhydrous ether. The pellet was collected for HPLC. HPLC analysis was done with a programmable Aglient Technology Zorbax 1200 series liquid chromatograph. The solvent system consisted of methanol (90\%) and water $(10 \%)$, at a flow rate of $1 \mathrm{ml}$ per minute; $0.1 \mathrm{mg}$ of cAMP solution per milliliter was eluted through the column (SB$\mathrm{C}_{18}, 5 \mu \mathrm{l}, 4.6 \times 250 \mathrm{~mm}$ ) and was detected at $259 \mathrm{~nm}$ UV. Each sample was eluted through the column in turn and peak values were detected with the same time as the standard.

\section{Measurement of the chitin content.}

Chitin (N-acetylglucosamine [GlcNAc]) content was analyzed as described (Bulik et al. 2003; Song et al. 2010). First, mycelial samples were freeze-dried, and then, $5 \mathrm{mg}$ of dried mycelia were resuspended in $1 \mathrm{ml}$ of $6 \% \mathrm{KOH}$ and were heated at $80^{\circ} \mathrm{C}$ for $90 \mathrm{~min}$. Samples were centrifuged $(16,000 \times g$, $10 \mathrm{~min}$ ) and pellets were washed with phosphate buffered saline in three cycles of centrifugation and suspension $(16,000 \times g$, $10 \mathrm{~min}$ ) before final suspension in $0.5 \mathrm{ml}$ of McIlvaine's buffer (pH 6). An aliquot of $100 \mathrm{ml}$ (13 units) of Streptomyces plicatus chitinase (Sigma) was added and was incubated for $16 \mathrm{~h}$ at $37^{\circ} \mathrm{C}$ with gentle mixing. Samples $(100 \mathrm{ml})$ were then combined with $100 \mathrm{ml}$ of $0.27 \mathrm{M}$ sodium borate $(\mathrm{pH} 9)$, were heated for $10 \mathrm{~min}$ at $100^{\circ} \mathrm{C}$, and finally, $1 \mathrm{ml}$ of freshly diluted (1:10) Ehrlich's reagent $(10 \mathrm{~g}$ p-dimethylaminobenzaldehyde in $1.25 \mathrm{ml}$ of $\mathrm{HCl}$ and $8.75 \mathrm{ml}$ of glacial acetic acid) was added. After incubating at $37^{\circ} \mathrm{C}$ for $20 \mathrm{~min}, 1 \mathrm{ml}$ of the sample was transferred to a $2.5-\mathrm{ml}$ plastic cuvette (Greiner) and the absorbance at $585 \mathrm{~nm}$ was recorded. Standard curves were prepared with GlcNAc (Sigma). The experiment was repeated three times.

\section{Western blot analysis of protein phosphorylation.}

The mutant and Guy11 strains were cultured in liquid CM media for 2 days. The intensity of the signal corresponding to phosphorylated Mps1 was detected by binding of an antiphosphop44/42 MAPK antibody (Cell Signaling Technology) and the Mpk1 antibody (N-terminal anti-Mpk1) from Santa Cruz Biotechnology was used as a control. Each experiment was repeated three times.

\section{ACKNOWLEDGMENTS}

This research was supported by the key program of Natural Science Foundation of China (Grant number 31530063 to Z. Zhang), National Science Foundation for Distinguished Young Scholars of China (Grant number 31325022 to Z. Zhang), Natural Science Foundation of China (Grant number 31271998 to Z. Zhang), and the especially appointed professorship (Jiangsu, China).

\section{LITERATURE CITED}

Alessi, D. R., Smythe, C., and Keyse, S. M. 1993. The human CL100 gene encodes a Tyr/Thr-protein phosphatase which potently and specifically inactivates MAP kinase and suppresses its activation by oncogenic ras in Xenopus oocyte extracts. Oncogene 8:2015-2020.

Alvarez, M. E., Pennell, R. I., Meijer, P. J., Ishikawa, A., Dixon, R. A., and Lamb, C. 1998. Reactive oxygen intermediates mediate a systemic signal network in the establishment of plant immunity. Cell 92:773-784.

Aroca, P., Bottaro, D. P., Ishibashi, T., Aaronson, S. A., and Santos, E. 1995. Human dual specificity phosphatase VHR activates maturation promotion factor and triggers meiotic maturation in Xenopus oocytes. J. Biol. Chem. 270:14229-14234.

Beeser, A. E., and Cooper, T. G. 1999. The dual-specificity protein phosphatase Yvhlp acts upstream of the protein kinase mcklp in promoting spore development in Saccharomyces cerevisiae. J. Bacteriol. 181:5219-5224.

Beeser, A. E., and Cooper, T. G. 2000. The dual-specificity protein phosphatase Yvh1p regulates sporulation, growth, and glycogen accumulation independently of catalytic activity in Saccharomyces cerevisiae via the cyclic AMP-dependent protein kinase cascade. J. Bacteriol. 182: 3517-3528.

Bolwell, G. P., Davies, D. R., Gerrish, C., Auh, C. K., and Murphy, T. M 1998. Comparative biochemistry of the oxidative burst produced by rose and french bean cells reveals two distinct mechanisms. Plant Physiol. 116:1379-1385.

Bulik, D. A., Olczak, M., Lucero, H. A., Osmond, B. C., Robbins, P. W., and Specht, C. A. 2003. Chitin synthesis in Saccharomyces cerevisiae in response to supplementation of growth medium with glucosamine and cell wall stress. Eukaryot. Cell 2:886-900.

Chen, Y., Zhai, S., Zhang, H., Zuo, R., Wang, J., Guo, M., Zheng, X., Wang, P., and Zhang, Z. 2014a. Shared and distinct functions of two Gti1/Pac2 family proteins in growth, morphogenesis and pathogenicity of Magnaporthe oryzae. Environ. Microbiol. 16:788-801.

Chen, Y., Zuo, R., Zhu, Q., Sun, Y., Li, M., Dong, Y., Ru, Y., Zhang, H., Zheng, X., and Zhang, Z. 2014b. MoLys2 is necessary for growth, conidiogenesis, lysine biosynthesis, and pathogenicity in Magnaporthe oryzae. Fungal Genet. Biol. 67:51-57. 
Chi, M. H., Park, S. Y., Kim, S., and Lee, Y. H. 2009. A novel pathogenicity gene is required in the rice blast fungus to suppress the basal defenses of the host. PLoS Pathog. 5:e1000401.

Daniel, P. B., Walker, W. H., and Habener, J. F. 1998. Cyclic AMP signaling and gene regulation. Annu. Rev. Nutr. 18:353-383.

Denu, J. M., and Dixon, J. E. 1995. A catalytic mechanism for the dualspecific phosphatases. Proc. Natl. Acad. Sci. U.S.A. 92:5910-5914.

Dixon, K. P., Xu, J. R., Smirnoff, N., and Talbot, N. J. 1999. Independent signaling pathways regulate cellular turgor during hyperosmotic stress and appressorium-mediated plant infection by Magnaporthe grisea. PLoS Pathog. 11:2045-2058.

Doi, K., Gartner, A., Ammerer, G., Errede, B., Shinkawa, H., Sugimoto, K., and Matsumoto, K. 1994. MSG5, a novel protein phosphatase promotes adaptation to pheromone response in S. cerevisiae. EMBO (Eur. Mol. Biol. Organ.) J. 13:61-70.

Dong, Y., Li, Y., Zhao, M., Jing, M., Liu, X., Liu, M., Guo, X., Zhang, X., Chen, Y., Liu, Y., Liu, Y., Ye, W., Zhang, H., Wang, Y., Zheng, X., Wang, P., and Zhang, Z. 2015. Global genome and transcriptome analyses of Magnaporthe oryzae epidemic isolate 98-06 uncover novel effectors and pathogenicity-related genes, revealing gene gain and lose dynamics in genome evolution. PLoS Pathog. 11:e1004801.

Egan, M. J., Wang, Z. Y., Jones, M. A., Smirnoff, N., and Talbot, N. J. 2007. Generation of reactive oxygen species by fungal NADPH oxidases is required for rice blast disease. Proc. Natl. Acad. Sci. U.S.A. 104:1177211777.

Fang, S., Lorick, K. L., Jensen, J. P., and Weissman, A. M. 2003. RING finger ubiquitin protein ligases: Implications for tumorigenesis, metastasis and for molecular targets in cancer. Semin. Cancer Biol. 13:5-14.

Gechev, T. S., and Hille, J. 2005. Hydrogen peroxide as a signal controlling plant programmed cell death. J. Cell Biol. 168:17-20.

Gordon, J. A. 1991. Use of vanadate as protein-phosphotyrosine phosphatase inhibitor. Methods Enzymol. 201:477-482.

Grant, M., Brown, I., Adams, S., Knight, M., Ainslie, A., and Mansfield, J. 2000. The RPM1 plant disease resistance gene facilitates a rapid and sustained increase in cytosolic calcium that is necessary for the oxidative burst and hypersensitive cell death. Plant J. 23:441-450.

Guan, K., Hakes, D. J., Wang, Y., Park, H. D., Cooper, T. G., and Dixon, J. E. 1992. A yeast protein phosphatase related to the vaccinia virus VH1 phosphatase is induced by nitrogen starvation. Proc. Natl. Acad. Sci. U.S.A. 89:12175-12179.

Guo, M., Guo, W., Chen, Y., Dong, S., Zhang, X., Zhang, H., Song, W., Wang, W., Wang, Q., Lv, R., Zhang, Z., Wang, Y., and Zheng, X. 2010. The basic leucine zipper transcription factor Moatf1 mediates oxidative stress responses and is necessary for full virulence of the rice blast fungus Magnaporthe oryzae. Mol. Plant-Microbe Interact 23:1053-1068.

Guo, M., Chen, Y., Du, Y., Dong, Y., Guo, W., Zhai, S., Zhang, H., Dong, S., Zhang, Z., Wang, Y., Wang, P., and Zheng, X. 2011. The bZIP transcription factor MoAP1 mediates the oxidative stress response and is critical for pathogenicity of the rice blast fungus Magnaporthe oryzae. PLoS Pathog. 7:e1001302.

Hanaoka, N., Umeyama, T., Ueno, K., Ueda, K., Beppu, T., Fugo, H., Uehara, Y., and Niimi, M. 2005. A putative dual-specific protein phosphatase encoded by YVH1 controls growth, filamentation and virulence in Candida albicans. Microbiology 151:2223-2232.

Harnpicharnchai, P., Jakovljevic, J., Horsey, E., Miles, T., Roman, J., Rout, M., Meagher, D., Imai, B., Guo, Y., Brame, C. J., Shabanowitz, J., Hunt, D. F., and Woolford, J. L., Jr. 2001. Composition and functional characterization of yeast 66S ribosome assembly intermediates. Mol. Cell 8:505-515.

Ishibashi, T., Bottaro, D. P., Chan, A., Miki, T., and Aaronson, S. A. 1992. Expression cloning of a human dual-specificity phosphatase. Proc. Natl. Acad. Sci. U.S.A. 89:12170-12174.

Jeon, J., Goh, J., Yoo, S., Chi, M. H., Choi, J., Rho, H. S., Park, J., Han, S. S., Kim, B. R., Park, S. Y., Kim, S., and Lee, Y. H. 2008. A putative MAP kinase kinase kinase, MCK1, is required for cell wall integrity and pathogenicity of the rice blast fungus, Magnaporthe oryzae. Mol. PlantMicrobe Interact 21:525-534.

Jones, J. D., and Dangl, J. L. 2006. The plant immune system. Nature 444: 323-329.

Kemmler, S., Occhipinti, L., Veisu, M., and Panse, V. G. 2009. Yvh1 is required for a late maturation step in the $60 \mathrm{~S}$ biogenesis pathway. J. Cell Biol. 186:863-880.

Keyse, S. M. 1995. An emerging family of dual specificity MAP kinase phosphatases. BBA 1265:152-160.

Kumar, R., Musiyenko, A., Cioffi, E., Oldenburg, A., Adams, B., Bitko, V., Krishna, S. S., and Barik, S. 2004. A zinc-binding dual-specificity YVH1 phosphatase in the malaria parasite, Plasmodium falciparum, and its interaction with the nuclear protein, pescadillo. Mol. Biochem. Parasitol. 133:297-310.
Lamb, C., and Dixon, R. A. 1997. The oxidative burst in plant disease resistance. Ann. Rev. Plant Physiol. Mol. Bio. 48:251-275.

Liu, Y., and Chang, A. 2009. A mutant plasma membrane protein is stabilized upon loss of Yvh1, a novel ribosome assembly factor. Genetics 181:907-915.

Liu, H., Suresh, A., Willard, F. S., Siderovski, D. P., Lu, S., and Naqvi, N. I. 2007. Rgs1 regulates multiple Galpha subunits in Magnaporthe pathogenesis, asexual growth and thigmotropism. EMBO (Eur. Mol. Biol. Organ.) J. 26:690-700.

Lo, K. Y., Li, Z., Wang, F., Marcotte, E. M., and Johnson, A. W. 2009. Ribosome stalk assembly requires the dual-specificity phosphatase Yvh1 for the exchange of Mrt4 with P0. J. Cell Biol. 186:849862.

Ma, P., Wera, S., Van Dijck, P., and Thevelein, J. M. 1999. The PDE1encoded low-affinity phosphodiesterase in the yeast Saccharomyces cerevisiae has a specific function in controlling agonist-induced cAMP signaling. Mol. Biol. Cell 10:91-104.

MacPherson, S., Larochelle, M., and Turcotte, B. 2006. A fungal family of transcriptional regulators: The zinc cluster proteins. Microbiol. Mol. Biol. Rep. 70:583-604.

Mellersh, D. G., Foulds, I. V., Higgins, V. J., and Heath, M. C. 2002. $\mathrm{H}_{2} \mathrm{O}_{2}$ plays different roles in determining penetration failure in three diverse plant-fungal interactions. Plant J. 29:257-268.

Muda, M., Manning, E. R., Orth, K., and Dixon, J. E. 1999. Identification of the human YVH1 protein-tyrosine phosphatase orthologue reveals a novel zinc binding domain essential for in vivo function. J. Biol. Chem. 274:23991-23995.

Nurnberger, T., Brunner, F., Kemmerling, B., and Piater, L. 2004. Innate immunity in plants and animals: Striking similarities and obvious differences. Immunol. Rev. 198:249-266.

Posner, B. I., Faure, R., Burgess, J. W., Bevan, A. P., Lachance, D., Zhang-Sun, G., Fantus, I. G., Ng, J. B., Hall, D. A., and Lum, B. S. 1994. Peroxovanadium compounds. A new class of potent phosphotyrosine phosphatase inhibitors which are insulin mimetics. J. Biol. Chem. 269:4596-4604.

Qi, Z., Wang, Q., Dou, X., Wang, W., Zhao, Q., Lv, R., Zhang, H., Zheng, X., Wang, P., and Zhang, Z. 2012. MoSwi6, an APSES family transcription factor, interacts with MoMps1 and is required for hyphal and conidial morphogenesis, appressorial function and pathogenicity of Magnaporthe oryzae. Mol. Plant Pathol. 13:677-689.

Qi, Z., Liu, M., Dong, Y., Zhu, Q., Li, L., Li, B., Yang, J., Li, Y., Ru, Y., Zhang, H., Zheng, X., Wang, P., and Zhang, Z. 2016. The syntaxin protein (MoSyn8) mediates intracellular trafficking to regulate conidiogenesis and pathogenicity of rice blast fungus. New Phytol. 209:16551667.

Ramanujam, R., and Naqvi, N. I. 2010. PdeH, a high-affinity cAMP phosphodiesterase, is a key regulator of asexual and pathogenic differentiation in Magnaporthe oryzae. PLoS Pathog. 6:e1000897.

Sacristán-Reviriego, A., Martin, H., and Molina, M. 2015. Identification of putative negative regulators of yeast signaling through a screening for protein phosphatases acting on cell wall integrity and mating MAPK pathways. Fungal Genet. Biol. 77:1-11.

Sakumoto, N., Mukai, Y., Uchida, K., Kouchi, T., Kuwajima, J., Nakagawa, Y., Sugioka, S., Yamamoto, E., Furuyama, T., Mizubuchi, H., Ohsugi, N., Sakuno, T., Kikuchi, K., Matsuoka, I., Ogawa, N., Kaneko, Y., and Harashima, S. 1999. A series of protein phosphatase gene disruptants in Saccharomyces cerevisiae. Yeast 15:1669-1679.

Song, W., Dou, X., Qi, Z., Wang, Q., Zhang, X., Zhang, H., Guo, M., Dong, S., Zhang, Z., Wang, P., and Zheng, X. 2010. R-SNARE homolog MoSec22 is required for conidiogenesis, cell wall integrity, and pathogenesis of Magnaporthe oryzae. PLoS One 5:e13193.

Sugiyama, M., Nugroho, S., Iida, N., Sakai, T., Kaneko, Y., and Harashima, S. 2011. Genetic interactions of ribosome maturation factors Yvh1 and Mrt4 influence mRNA decay, glycogen accumulation, and the expression of early meiotic genes in Saccharomyces cerevisiae. J. Biochem. 150: 103-111.

Sun, H., Charles, C. H., Lau, L. F., and Tonks, N. K. 1993. MKP-1 (3CH134), an immediate early gene product, is a dual specificity phosphatase that dephosphorylates MAP kinase in vivo. Cell 75:487-493.

Sweigard, J. A., Chumley, F. G., and Valent, B. 1992. Disruption of a Magnaporthe grisea cutinase gene. Mol. Gen. Genet. 232:183-190.

Talbot, N. J., Ebbole, D. J., and Hamer, J. E. 1993. Identification and characterization of MPG1, a gene involved in pathogenicity from the rice blast fungus Magnaporthe grisea. Plant Cell 5:1575-1590.

Tanabe, S., Nishizawa, Y., and Minami, E. 2009. Effects of catalase on the accumulation of $\mathrm{H} 2 \mathrm{O} 2 \mathrm{in}$ rice cells inoculated with rice blast fungus, Magnaporthe oryzae. Physiol. Plant. 137:148-154.

Wang, J., Du, Y., Zhang, H., Zhou, C., Qi, Z., Zheng, X., Wang, P., and Zhang, Z. 2013. The actin-regulating kinase homologue MoArk1 plays a 
pleiotropic function in Magnaporthe oryzae. Mol. Plant Pathol. 14:470482.

Weber, R. W., Pitt, D., and Webster, J. 1998. Teaching techniques for mycology: 3. Amylase secretion by Aspergillus oryzae. Mycologist 12: 8-9.

Wilson, R. A., and Talbot, N. J. 2009. Under pressure: Investigating the biology of plant infection by Magnaporthe oryzae. Nat. Rev. Microbiol. 7:185-195.

Wood, P. J., and Fulcher, R. G. 1983. Dye interactions. A basis for specific detection and histochemistry of polysaccharides. J. Histochem. Cytochem. 31:823-826.

Xu, J. R., Staiger, C. J., and Hamer, J. E. 1998. Inactivation of the mitogenactivated protein kinase Mps1 from the rice blast fungus prevents penetration of host cells but allows activation of plant defense responses. Proc. Natl. Acad. Sci. U.S.A. 95:12713-12718.

Yin, Z., Tang, W., Wang, J., Liu, X., Yang, L., Gao, C., Zhang, J., Zhang, H., Zheng, X., and Wang, P. 2015. Phosphodiesterase MoPdeH targets MoMck1 of the conserved mitogen-activated protein (MAP) kinase signaling pathway to regulate cell wall integrity in rice blast fungus Magnaporthe oryzae. Mol. Plant Pathol. doi:10.1111/mpp.12317. Published online.

Zhang, H., Fang, Q., Zhang, Z., Wang, Y., and Zheng, X. 2009a. The role of respiratory burst oxidase homologues in elicitor-induced stomatal closure and hypersensitive response in Nicotiana benthamiana. J. Exp. Bot. 60:3109-3122.
Zhang, H., Zhao, Q., Liu, K., Zhang, Z., Wang, Y., and Zheng, X. 2009b. MgCRZ1, a transcription factor of Magnaporthe grisea, controls growth, development and is involved in full virulence. FEMS Microbiol. Lett. 293:160-169.

Zhang, H., Liu, K., Zhang, X., Tang, W., Wang, J., Guo, M., Zhao, Q., Zheng, X., Wang, P., and Zhang, Z. 2011a. Two phosphodiesterase genes, PDEL and PDEH, regulate development and pathogenicity by modulating intracellular cyclic AMP levels in Magnaporthe oryzae. PLoS One 6:e17241.

Zhang, H., Tang, W., Liu, K., Huang, Q., Zhang, X., Yan, X., Chen, Y., Wang, J., Qi, Z., Wang, Z., Zheng, X., Wang, P., and Zhang, Z. 2011 b. Eight RGS and RGS-like proteins orchestrate growth, differentiation, and pathogenicity of Magnaporthe oryzae. PLoS Pathog. 7:e1002450.

Zolnierowicz, S., and Bollen, M. 2000. Protein phosphorylation and protein phosphatases. De Panne, Belgium, September 19-24, 1999. EMBO (Eur. Mol. Biol. Organ.) J. 19:483-488.

Zuk, D., Belk, J. P., and Jacobson, A. 1999. Temperature-sensitive mutations in the Saccharomyces cerevisiae MRT4, GRC5, SLA2 and THS1 genes result in defects in mRNA turnover. Genetics 153:35-47.

\section{AUTHOR-RECOMMENDED INTERNET RESOURCE}

Broad Institute Magnaporthe grisea database: http://www.broad.mit.edu/annotation/genome/magnaporthe_grisea/ Home.html 